\title{
Modulation of subsets of cardiac B lymphocytes improves cardiac function after acute injury
}

\author{
Luigi Adamo, ${ }^{1}$ Lora J. Staloch, ${ }^{1}$ Cibele Rocha-Resende, ${ }^{1}$ Scot J. Matkovich, ${ }^{1}$ Wenlong Jiang, ${ }^{1}$ \\ Geetika Bajpai,, ${ }^{1}$ Carla J. Weinheimer, ${ }^{1}$ Attila Kovacs, ${ }^{1}$ Joel D. Schilling, ${ }^{1}$ Philip M. Barger, ${ }^{1}$ \\ Deepta Bhattacharya, ${ }^{2}$ and Douglas L. Mann' \\ ${ }^{1}$ Cardiovascular Division, Department of Medicine, Washington University School of Medicine, St. Louis, Missouri, USA. \\ 2Department of Immunobiology, University of Arizona, Tucson, Arizona, USA.
}

\begin{abstract}
Despite the long-standing recognition that the immune response to acute myocardial injury contributes to adverse left ventricular (LV) remodeling, it has not been possible to effectively target this clinically. Using 2 different in vivo models of acute myocardial injury, we show that pirfenidone confers beneficial effects in the murine heart through an unexpected mechanism that depends on cardiac B lymphocytes. Naive hearts contained a large population of CD19+CD11b-CD23-CD21-IgD+IgM ${ }^{10}$ Iymphocytes, and 2 smaller populations of CD19+CD11b+ B1a and B1b cells. In response to tissue injury, there was an increase in neutrophils, monocytes, macrophages, as well as an increase in CD19+ CD11b- B lymphocytes. Treatment with pirfenidone had no effect on the number of neutrophils, monocytes, or macrophages, but decreased CD19+CD11b- lymphocytes. B cell depletion abrogated the beneficial effects of pirfenidone. In vitro studies demonstrated that stimulation with lipopolysaccharide and extracts from necrotic cells activated CD19+ lymphocytes through a TIRAP-dependent pathway. Treatment with pirfenidone attenuated this activation of $B$ cells. These findings reveal a previously unappreciated complexity of myocardial B lymphocytes within the inflammatory infiltrate triggered by cardiac injury and suggest that pirfenidone exerts beneficial effects in the heart through a unique mechanism that involves modulation of cardiac B lymphocytes.
\end{abstract}

Conflict of interest: The authors have declared that no conflict of interest exists.

Submitted: January 24, 2018 Accepted: April 24, 2018 Published: June 7, 2018

\section{Reference information:} JCI Insight. 2018;3(11):e120137. https://doi.org/10.1172/jci. insight.120137.

\section{Introduction}

Myocardial injury triggers a complex series of inflammatory responses that result in the recruitment and activation of cells of the innate and adaptive immune systems. This brisk inflammatory response upregulates a suite of cytoprotective and/or reparative responses that provide the heart with short-term adaptations to environmental stress (reviewed in ref. 1). However, this same inflammatory response often results in unintended collateral myocardial damage that can lead to adverse left ventricular (LV) remodeling and progressive dysfunction. Although there have been a number of clinical attempts to target inflammation following cardiac injury (reviewed in ref. 2), there are currently no FDA-approved immunomodulatory therapies for the treatment of acute myocardial injury.

Pirfenidone is an orally active pyridinone derivative whose mechanism of action is not fully understood. Pirfenidone has been shown to have antifibrotic, antiinflammatory, and antioxidant properties (3), and is currently FDA approved for the treatment of idiopathic pulmonary fibrosis. Pirfenidone has also been shown to be protective in the heart in a number of different experimental models of cardiac injury including transverse aortic constriction $(4,5)$, angiotensin II-induced cardiac hypertrophy (6), DOCA salt hypertensive cardiomyopathy (7), Duchenne muscular dystrophy-associated cardiomyopathy (8), diabetes-associated cardiomyopathy (9), tachycardia-induced cardiomyopathy (10), and myocardial infarction $(11,12)$. While the majority of the cardioprotective effects of pirfenidone have been attributed to a reduction in cardiac fibrosis, the precise cellular target and mechanism of action for the cardioprotective effects of pirfenidone in vivo remain unknown. Noting that pirfenidone has been shown to have a number of different immunomodulatory properties that are relevant to immune-mediated collateral damage, we sought to determine whether pirfenidone would favorably modulate the 
inflammatory response following acute cardiac injury. Here we show in 2 different models of cardiac injury that unexpectedly pirfenidone exerts salutary effects through a potentially novel mechanism that involves the modulation of distinct B lymphocyte populations in the heart.

\section{Results}

Pirfenidone improves survival after diphtheria toxin-mediated acute myocardial injury. To determine the effects of pirfenidone on acute myocardial injury we employed the diphtheria toxin (DT) cardiac myocyte cell ablation model, as previously described (13). Adult Rosa26-DT ${ }^{\text {Mlc2v-Cre }}$ mice injected with DT develop LV dysfunction and remodeling, and associated increased mortality within 2 weeks (13). As shown in Figure 1A, the survival of pirfenidone-treated mice was significantly improved when compared with untreated, DT-treated littermate controls $(P=0.03)$. The protective effect of pirfenidone was not secondary to a significant attenuation in DT-induced cardiac myocyte cell death, insofar as there were no significant differences in serum troponin levels $(P=0.34$, Figure 1B), the prevalence of cardiac myocyte apoptosis $(P=0.39$, Figure $1 \mathrm{C})$, and extent of Evans blue dye uptake $(P=0.26$, Figure 1D) between the mice fed normal chow and mice fed chow with pirfenidone.

Pirfenidone reduces cardiac $C D 19^{+} B$ lymphocytes following DT-mediated acute myocardial injury. Given that treatment with pirfenidone did not reduce cardiac myocyte necrosis or apoptosis, we asked whether pirfenidone improved survival by modulating the innate immune response to acute cardiac injury. Accordingly, we performed FACS analysis 4 days after DT injection. The gating strategy for this FACS analysis is shown in Supplemental Figure 1A (supplemental material available online with this article; https://doi. org/10.1172/jci.insight.120137DS1). In preliminary control studies, we determined that treatment with pirfenidone for 1 week in naive WT hearts had no significant effect on the number of $\mathrm{CD} 45^{+}$cells $/ \mathrm{mg}$ tissue $(P=0.53)$, Ly6G ${ }^{+}$neutrophils $(P=0.82)$, Ly6 $\mathrm{C}^{+} \mathrm{CD}^{4} 4^{1 \mathrm{o} /}-$ monocytes $(P=0.81), \mathrm{CD}^{+} 4^{+} \mathrm{Ly}^{6} \mathrm{C}^{\mathrm{lo} /-}$ macrophages $(P=0.82)$, or $\mathrm{CD} 19^{+}$B lymphocytes $(P=0.94$; Supplemental Figure $1, \mathrm{~B}$ and $\mathrm{C})$.

As shown in Figure 2, there were no significant differences in the DT-injured hearts from mice treated with pirfenidone chow or normal chow with respect to the number of myocardial CD45 ${ }^{+}$cells $(P=0.8$, Figure 2A), Ly6G ${ }^{+}$neutrophils $(P=0.27$, Figure $2 \mathrm{~B})$, Ly6 $\mathrm{C}^{+} \mathrm{CD} 64^{\mathrm{lo} /-}$ monocytes $(P=0.15$, Figure $2 \mathrm{~B})$, and Ly6 $\mathrm{C}^{\mathrm{lo} /-} \mathrm{CD} 64^{+}$macrophages $(P=0.9$, Figure $2 \mathrm{~B})$. The adult heart macrophage pool consists of resident and recruited cells, the latter of which have been associated with adverse LV remodeling following injury. These subpopulations are largely divided by the expression of CCR2 and MHC-II $(13,14)$. Therefore, we further characterized the macrophage populations in control and pirfenidone-treated animals. As shown in Figure 2, C and D there was no significant difference in the percentage of MHC-II ${ }^{\text {hi }} \mathrm{CCR} 2^{\text {lo }}(P=0.43)$, $\mathrm{MHC}-\mathrm{II}^{\mathrm{hi}} \mathrm{CCR} 2^{\text {hi }}(P=0.36)$, MHC-II ${ }^{\mathrm{lo}} \mathrm{CCR} 2^{\text {hi }}(P=0.21)$, or $\mathrm{MHC}-\mathrm{II}^{\mathrm{lo}} \mathrm{CCR} 2^{\text {lo }}(P=0.11)$ macrophage subsets in the presence and absence of treatment with pirfenidone. Despite the lack of differences in cardiac myeloid populations after damage, we did observe that treatment with pirfenidone resulted in a greater than 3-fold reduction in the percentage of $\mathrm{CD} 19^{+}$myocardial B lymphocytes following DT-induced injury $(P=$ 0.02 , Figure $2 \mathrm{~B}$ ) when compared with mice that were fed normal chow.

Pirfenidone reduces adverse $L V$ remodeling and cardiac $C D 19^{+} B$ lymphocytes after closed-chest ischemia-reperfusion injury. To determine the effects of pirfenidone in a more pathophysiologically relevant model of cardiac injury, we subjected control mice and pirfenidone-treated mice to closed-chest ischemia-reperfusion (I/R) injury (90 minutes of ischemia), as previously described (15). As shown in Figure 3A, there was no difference $(P=$ $0.68)$ in the area at risk in control and pirfenidone-treated animals, as determined by the segmental wall motion score index (SWMSI). Treatment with pirfenidone resulted in a significant decrease in the heart weight-to-tibia length ratio (Figure 3, B and C) and LV mass measured by 2-D echocardiography $(P=0.03$, Figure 3D) when compared with control mice. Consistent with these findings, we observed a significant $(P=0.02)$ decrease in LV end-diastolic volumes (Figure $3 \mathrm{E}$ ) in pirfenidone-treated animals when compared with control animals, whereas there was a trend $(P=0.08)$ towards improvement in LV ejection fraction (LVEF) in the treatment group (Figure 3F). Figure 3G shows representative Masson's trichrome staining in control and pirfenidone-treated animals, whereas Figure $3 \mathrm{H}$ summarizes the results of the group data. As shown in Figure $3 \mathrm{H}$, there was no significant difference $(P=0.4)$ in the percentage of myocardium exhibiting collagen staining with Masson's at 2 weeks, when the pirfenidone chow mice were compared to the mice fed normal chow.

As the preceding experiments examined the effect of pretreatment with pirfenidone prior to I/R injury, we sought to determine whether the acute administration of pirfenidone was sufficient to influence LV remodeling after I/R injury. We therefore treated mice with intravenous pirfenidone or diluent soon after reperfusion injury, followed by i.p. injections for 1 day and oral pirfenidone in the chow for 14 days. Supple- 


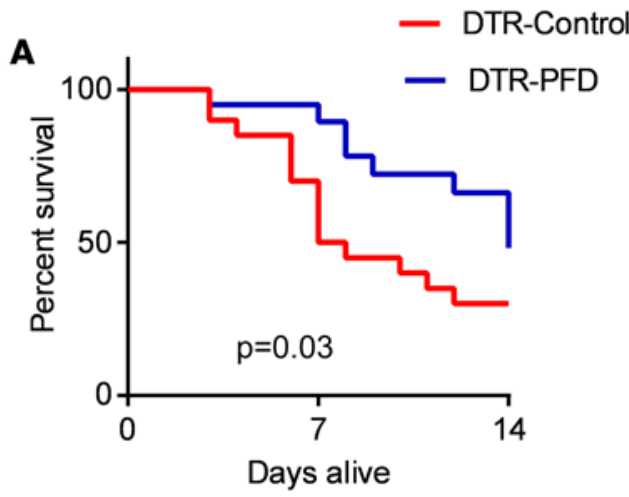

B

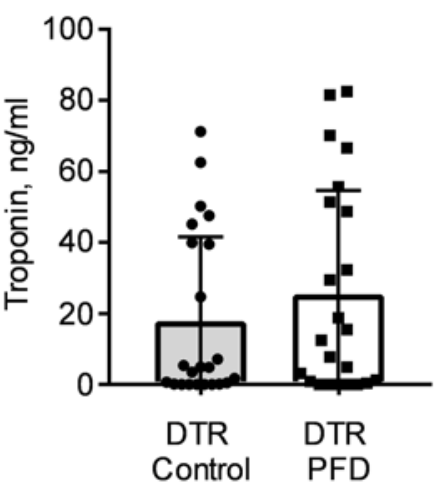

C

\section{DTR-Control}
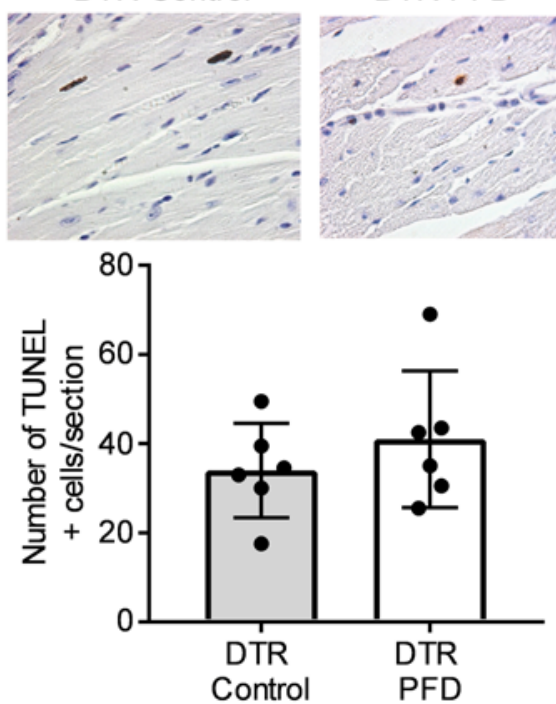

DTR-PFD

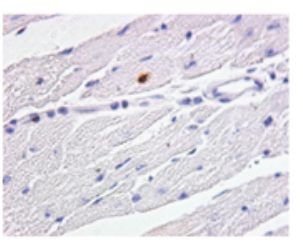

PFD
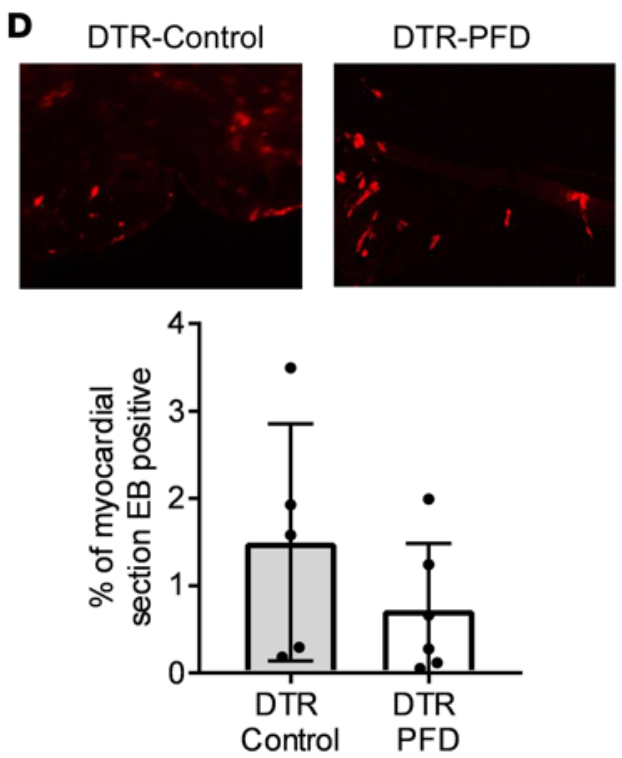

Figure 1. Effect of pirfenidone on mortality and cardiac myocyte cell death after DT treatment. Mice expressing the diphtheria toxin receptor (DTR) in the myocardium were exposed to diphtheria toxin (DT) and fed either chow enriched with pirfenidone (DTR-PFD) or regular chow (DTR-control). (A) Kaplan-Meier survival curves of DTR control and DTRPFD mice ( $n=20$ per group). (B) Serum troponin levels measured at day 4 after DT treatment in DTR-PFD and DTR-control animals ( $n=23$ /group). (C) Cardiac myocyte apoptosis measured at day 4 after treatment with DT. Upper panels are representative histological sections of myocardium from DTR-control and DTR-PFD mice at $\times 40$ magnification. Lower panel summarizes the group data ( $n=6$ mice/group, 4 sections per animal analyzed). (D) Evans blue (EB) dye uptake at day 4 after DT treatment in DTR-control and DTR-PFD animals; upper panels are representative fluorescence microscopy images at $\times 10$ magnification; lower panel summarizes the group data ( $n=5$ control; $n=6$ mice with pirfenidone; 4 sections per animal analyzed). Bars represent the mean, and error bars represent standard deviation. $P$ values were calculated with the Gehan-Breslow-Wilcoxon method for panel A and with Student's $t$ test for panels B-D.

mental Figure 2 shows that the area at risk was not different $(P=0.47)$ in the control and pirfenidone-treated animals. However, the salient finding shown by Supplemental Figure 2 is that acute administration of pirfenidone significantly $(P=0.015)$ reduced the heart weight/tibia length ratio $(P=0.015$, Supplemental Figure $2 \mathrm{~B}), \mathrm{LV}$ mass by 2 -D echocardiography $(P=0.029$, Supplemental Figure $2 \mathrm{D})$, and LV end-diastolic volume $(P=0.037$, Supplemental Figure $2 \mathrm{E})$, when compared with diluent-treated control mice.

To determine whether the mechanism(s) for the beneficial effects of pirfenidone in the closed-chest I/R injury model was dependent on modulation of the innate immune response to acute cardiac injury, we harvested hearts on day 4 after I/R injury, and analyzed the inflammatory infiltrate via FACS. Consistent with the results observed with the DT-induced injury model, treatment with pirfenidone did not significantly $(P=0.98$, Figure $4 \mathrm{~A})$ alter the total number of $\mathrm{CD} 45^{+}$cells per $\mathrm{mg}$ heart, $\mathrm{Ly}_{6 \mathrm{G}}{ }^{+}$neutrophils $(P=$ 0.44 , Figure $4 \mathrm{~B})$, Ly6 $\mathrm{C}^{+} \mathrm{CD} 64^{\mathrm{lo} /}-$ monocytes $(P=0.74$, Figure $4 \mathrm{~B})$, or $\mathrm{CD} 64^{+} \mathrm{Ly} 6 \mathrm{C}^{\mathrm{lo} /-}$ macrophages $(P=$ 0.91 , Figure 4B). Moreover, treatment with pirfenidone did not alter the relative prevalence of macrophage 

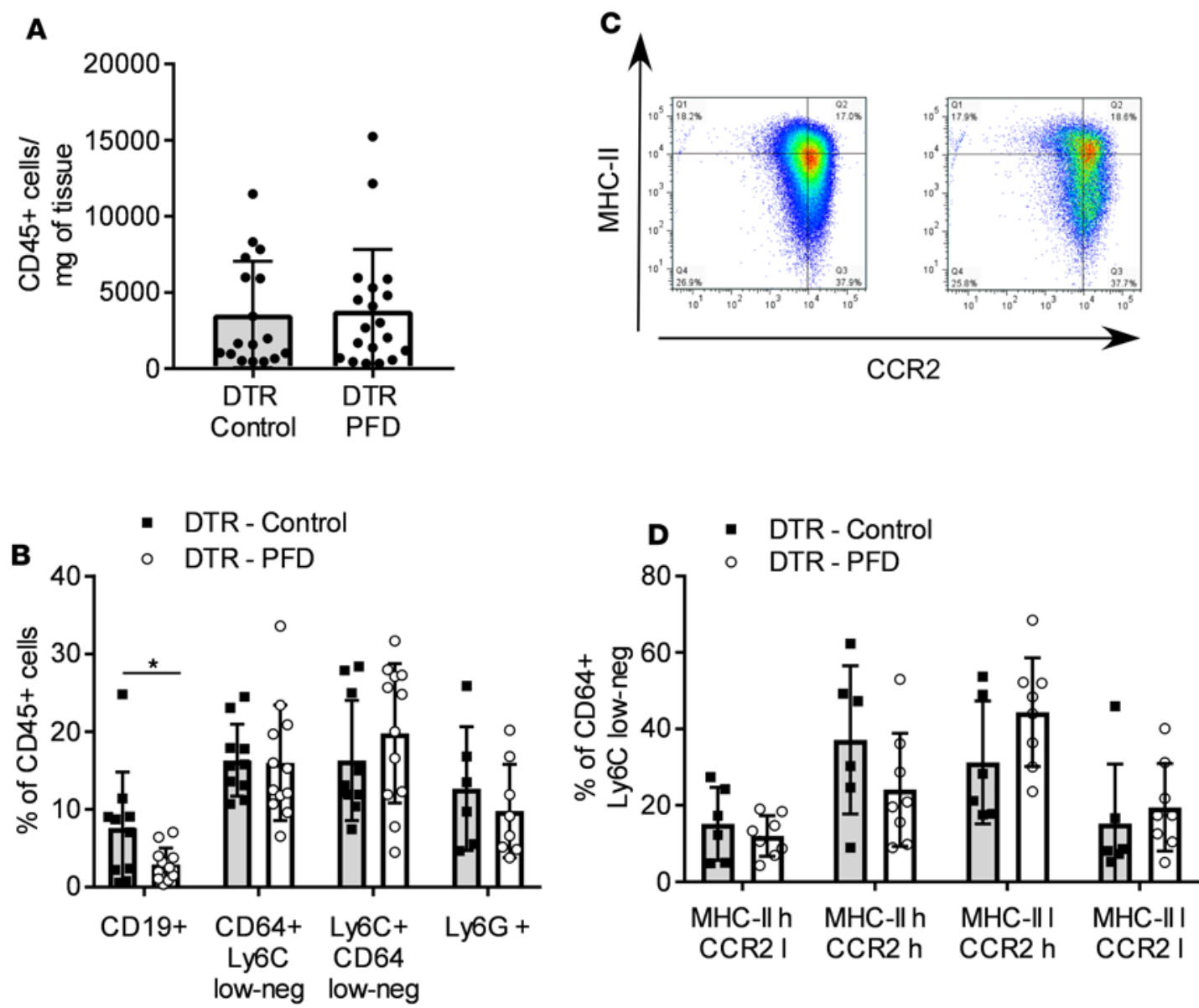

Figure 2. Effect of pirfenidone on myocardial inflammation (day 4) after DT treatment. Mice expressing the diphtheria toxin receptor (DTR) in the myocardium were exposed to diphtheria toxin (DT) and fed either chow enriched with pirfenidone (DTRPFD) or regular chow (DTR-control). Mice were sacrificed at day 4 after DT injection and the heart was collected for analysis via flow cytometry. (A) Total number of $C D 45^{+}$cells/mg heart tissue ( $n=17$ control, $n=19$ pirfenidone). (B) Leukocyte subsets in the myocardium (percentage of total: $\mathrm{CD}^{9} 9^{+}, n=14$ control, $n=16$ pirfenidone; Ly6g,$n=6 /$ group, Ly6C+CD64 ${ }^{10 /}, n=10$ control, $n$ $=12$ pirfenidone; $\mathrm{CD}_{4}{ }^{+}$Ly6 $\mathrm{C}^{\mathrm{lo} /-}, n=10$ control, $n=12$ pirfenidone). (C) Representative FACS analysis of MHC-II and CCR-2 macrophages and monocytes. (D) Macrophage/monocyte subsets in the myocardium as defined by expression of CCR2, low (I) or high (h) and MHC-II expression, low (I) or high (h). Percentage of total, $n=10$ control, $n=12$ pirfenidone. ${ }^{*} P<0.05$. Bars represent the mean, and error bars represent standard deviation. $P$ values were calculated with Student's $t$ test.

subpopulations as defined by levels of expression of CCR2 and MHC-II (Figure 4, C and D). However, we again noticed that treatment with pirfenidone significantly $(P=0.004)$ reduced the percentage of $\mathrm{CD} 19^{+} \mathrm{B}$ lymphocytes (Figure 4B) in the heart following injury.

Pirfenidone modulates subsets of cardiac B lymphocytes following cardiac injury. To further interrogate the effects of pirfenidone on cardiac B lymphocytes, we first analyzed the surface antigens of cardiac B cells using known markers of B lymphocytic subtypes. The gating strategy that was used is illustrated in Supplemental Figure 3 and staining of splenic B lymphocytes is provided for reference in Supplemental Figure 4. As shown in Figure 5 , the majority of $\mathrm{CD} 19^{+}$cardiac $\mathrm{B}$ cells in the uninjured adult heart were $\mathrm{CD} 19^{+} \mathrm{CD} 11 \mathrm{~b}$. Further characterization of this population revealed that they were $\mathrm{IgD}^{+}$and $\mathrm{IgM}^{10 /-} \mathrm{CD} 21^{-} \mathrm{CD} 23^{-} \mathrm{CD} 11 \mathrm{c}^{-}$(Supplemental Figure 3). Approximately $10 \%$ of the resident cardiac $B$ cells in uninjured hearts were $C D 19^{+} C D 11 b^{+}$. This population of cells was predominately $\mathrm{CD} 5 \mathrm{IgM}^{+}$, which is characteristic of $\mathrm{B} 1 \mathrm{~b}$ lymphocytes, whereas a smaller percentage was $\mathrm{CD}^{+} \mathrm{IgM}^{+}$, which is characteristic of B1a lymphocytes (16) (Figure $5 \mathrm{~A}$ ).

We next analyzed B cell profiles in the presence or absence of pirfenidone in the DT-induced injury model and the closed-chest I/R injury model. As shown in Figure 5, in both models of cardiac injury, the relative ratio of $\mathrm{CD} 19^{+} \mathrm{CD} 11 \mathrm{~b}^{-}$cells to $\mathrm{B} 1$ cells on day 4 after cardiac injury remained unchanged relative to naive hearts. In the DT-injured hearts (day 4) there was a small but nonsignificant (Figure 5B) increase 
A
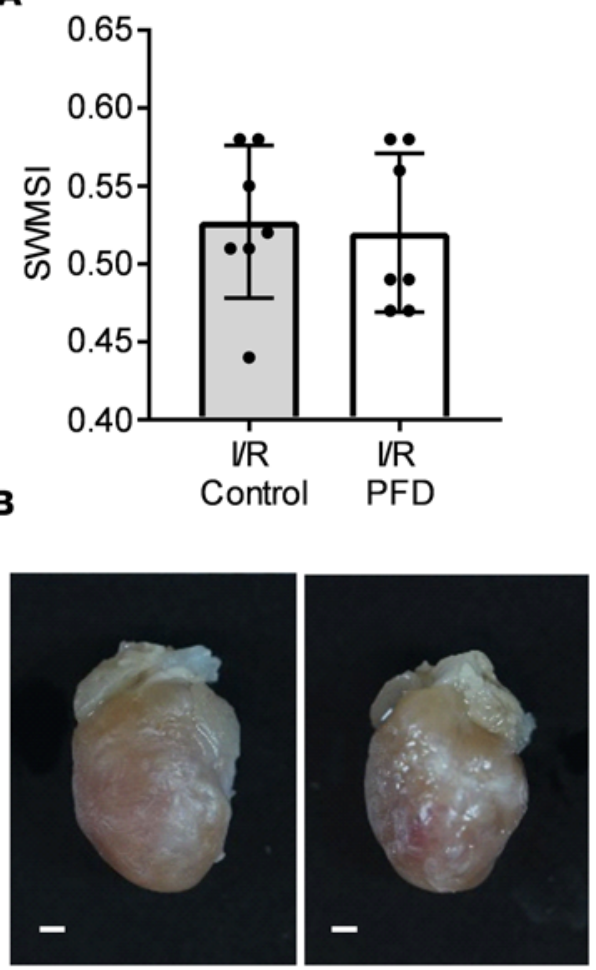

C
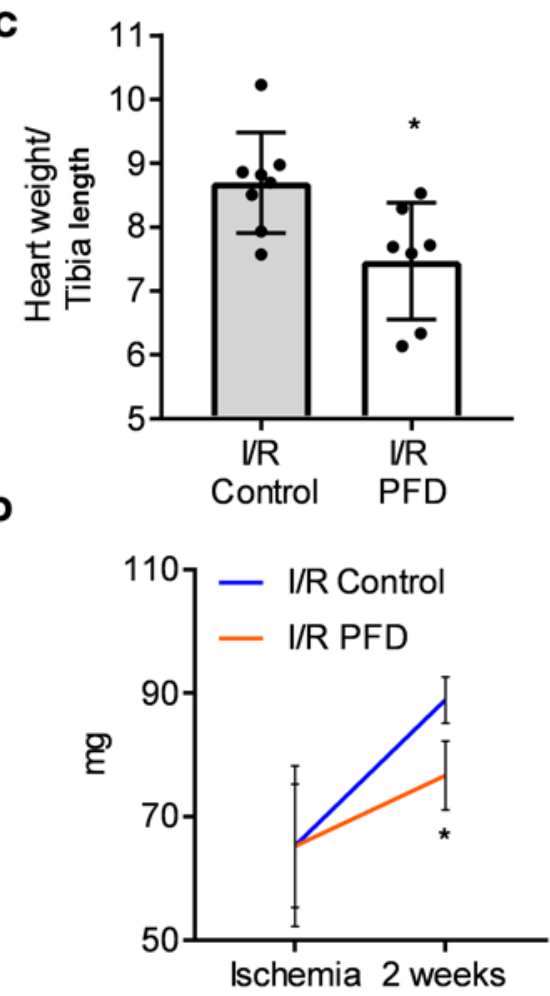

E

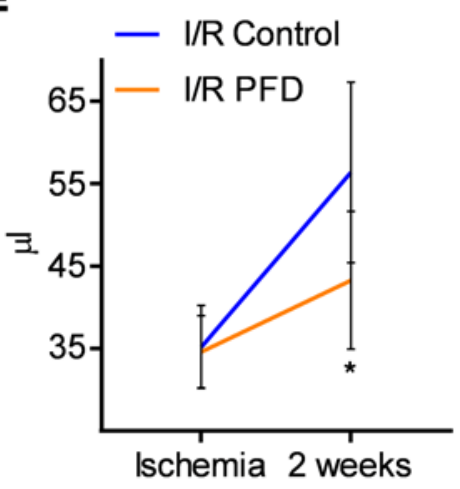

$\mathbf{F}$

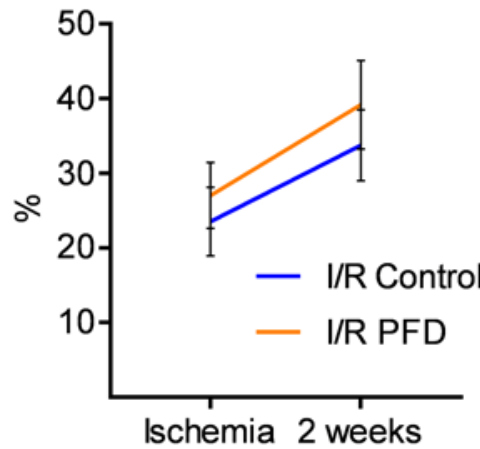

G

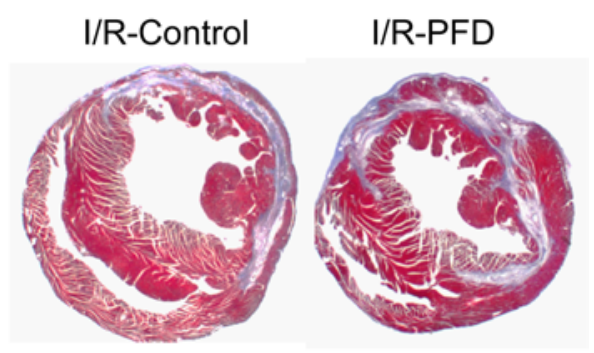

H

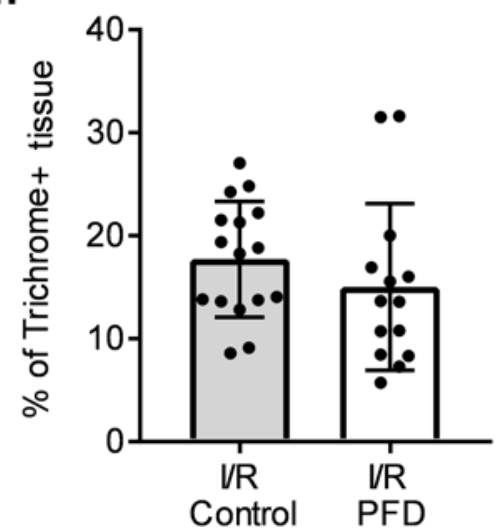

Figure 3. Effect of pirfenidone on LV structure and function after closed-chest I/R injury. Wild-type mice were subjected to 90 minutes of closed-chest ischemia followed by 2 weeks of reperfusion (I/R injury). Mice were fed either chow enriched with pirfenidone or regular chow. (A) Area at risk during closed-chest ischemia as determined by the simplified segmental wall motion score index (SWMSI) at time of ischemia. (B) Representative pictures of hearts harvested from control mice (left) and pirfenidone-treated animals (right) 2 weeks after I/R injury. Scale bar: $1 \mathrm{~mm}$. (C) Gravimetric analysis of hearts harvested from control mice (I/R-control) and pirfenidone-treated animals (I/R-PFD). $n=8$ control, $n=7$ pirfenidone. (D-F)

Echocardiographic assessment of myocardial function at the time of ischemia and 2 weeks after I/R injury. $n=8 \mathrm{I} / \mathrm{R}$-control, $n=7$ I/R-PFD. (D) Left ventricular (LV) mass (LVM) by 2-D echocardiography. (E) LV end-diastolic volume (LVEDV). (F) LV ejection fraction (LVEF). (C) Representative trichrome staining of histological sections of hearts from control animals (left panel) and pirfenidone-treated animals (right panel). Original magnification, $\times 1.25$. (H) Quantitative assessment of the percentage trichrome-positive staining, 2 sections analyzed per heart. $n$ $=16$ sections $\mathrm{I} / \mathrm{R}$-control, $n=$ 14 sections I/R-PFD. ${ }^{*} P<0.05$. Bars represent the mean, and error bars represent standard deviation. $P$ values were calculated with Student's $t$ test. 


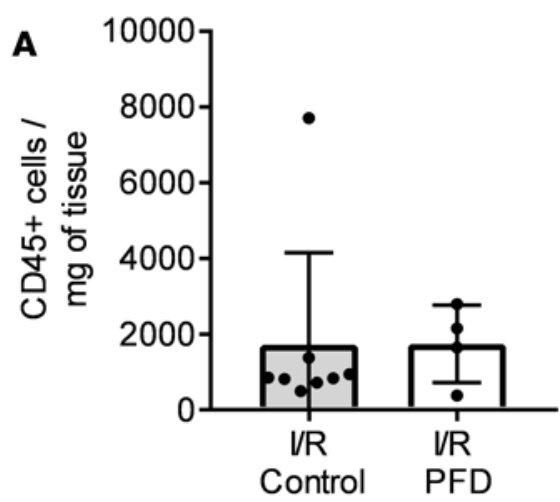

B

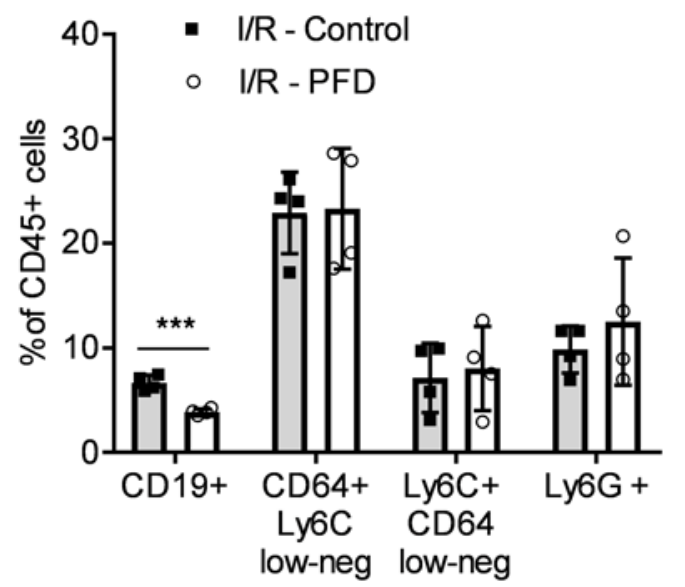

C

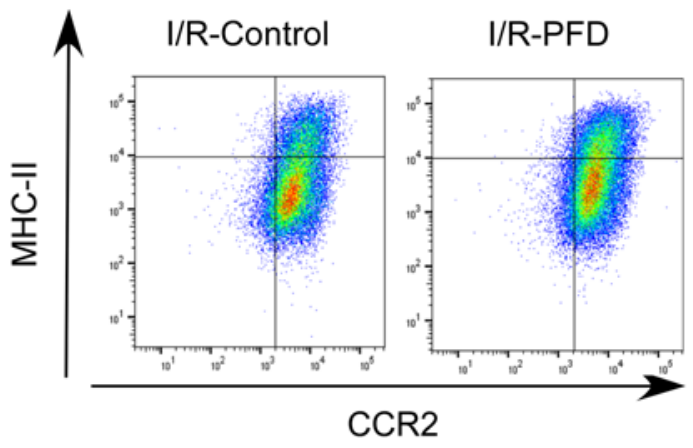

D

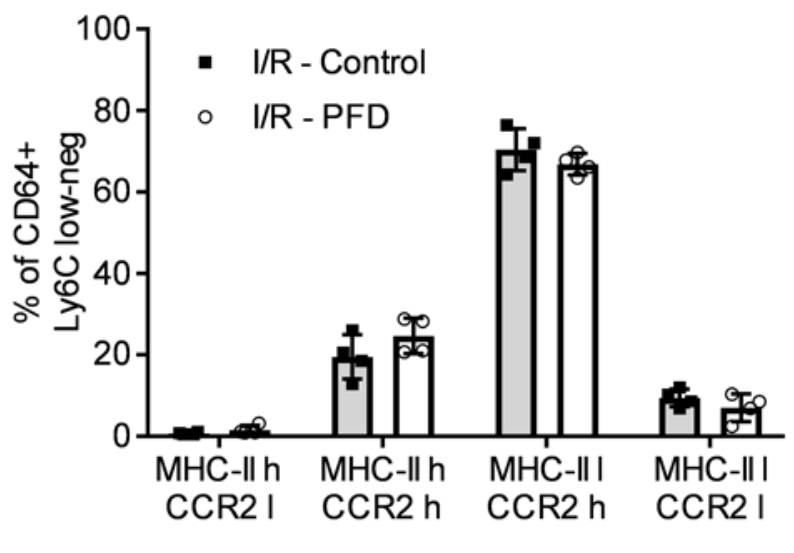

Figure 4. Effect of pirfenidone on myocardial inflammation (day 4) after I/R injury. Wild-type mice were subjected to 90 minutes closed-chest ischemia followed by reperfusion (I/R injury). Mice were fed either chow enriched with pirfenidone (PFD) or regular chow. Mice were sacrificed at day 4 after I/R injury and the heart was collected for analysis via flow cytometry. $n=8 \mathrm{I} / \mathrm{R}$-control, $n=4$ I/R-PFD. (A) Total number of CD45+ cells/mg heart tissue. (B) Leukocyte subsets in the myocardium (percentage of total: $\mathrm{CD19}^{+}, \mathrm{Ly6g}^{+}$, Ly6C+CD64 ${ }^{10 /-}$, CD64+Ly6C ${ }^{\mathrm{lo}} ; n=4$ /group). (C) Representative FACS analysis of MHC-II and CCR-2 macrophages and monocytes. (D) Macrophage/monocyte subsets in the myocardium as defined by expression of CCR 2, low (I) or high (h) and MHC-II expression, low (I) or high (h). Percentage of total, $n=4$ /group.*** $P<0.001$. Bars represent the mean, and error bars represent standard deviation. $P$ values were calculated with Student's $t$ test.

in the number of $\mathrm{CD} 19^{+}$cells $/ \mathrm{mg}$ of cardiac tissue $(P=0.09)$ and $\mathrm{CD} 19^{+} \mathrm{CD} 11 \mathrm{~b}^{+}$cells $/ \mathrm{mg}$ of cardiac tissue $(P=0.14)$, but no change in the number of $\mathrm{CD} 19^{+} \mathrm{CD} 11 \mathrm{~b}^{-}$cells $/ \mathrm{mg}$ of cardiac tissue $(P=0.96)$ when compared with naive hearts. In contrast, there was a significant 2-fold increase in $\mathrm{CD}^{+} 9^{+}(P<0.001)$ and $\mathrm{CD} 19^{+} \mathrm{CD} 11 \mathrm{~b}^{-}(P<0.001)$ cells at the same time point following I/R injury (Figure 5C). In the DT-induced injury cardiac myocyte ablation model, the pirfenidone-treated animals had significantly fewer $(P<$ $0.01) \mathrm{CD} 9^{+}$cells/mg of tissue, with a significant $(P=0.04)$ reduction in the subset of $\mathrm{CD} 19^{+} \mathrm{CD} 11 \mathrm{~b}^{-}$cells (Figure $5 \mathrm{~B}$ ). We observed very similar changes in the closed-chest I/R injury model, in which pirfenidone treatment resulted in a significant $(P<0.001)$ reduction in $\mathrm{CD} 19^{+}$lymphocytes on day 4 after $\mathrm{I} / \mathrm{R}$, as well as a significant $(P<0.001)$ reduction in the subset of $\mathrm{CD} 19^{+} \mathrm{CD} 11 \mathrm{~b}^{-} \mathrm{B}$ lymphocytes (Figure $5 \mathrm{C}$ ).

The cardioprotective effect of pirfenidone is mediated by $B$ lymphocytes. Viewed together, the above results suggest that the cardioprotective effects of pirfenidone may be mediated, at least in part, by cardiac B lymphocytes. To test this hypothesis, we depleted native B cells using a commercially available anti-CD20 antibody, and then repeated the closed-chest I/R studies in the presence and absence of pirfenidone. Given that the mice were instrumented 7 days prior to I/R injury in the closed-chest model, we injected the mice with anti-CD20 antibody at the time of instrumentation. The efficiency of anti-CD20 antibody B cell depletion was assessed 8 days after anti-CD20 injection (i.e., at the time that I/R injury is performed) and on day 12 after injection (i.e., day 4 after I/R). As shown in Supplemental Figure 5, A and B, a single injection of anti-CD20 antibody ablated over $99 \%$ of splenic B cells and cardiac B cells by day 8 . We noticed that 


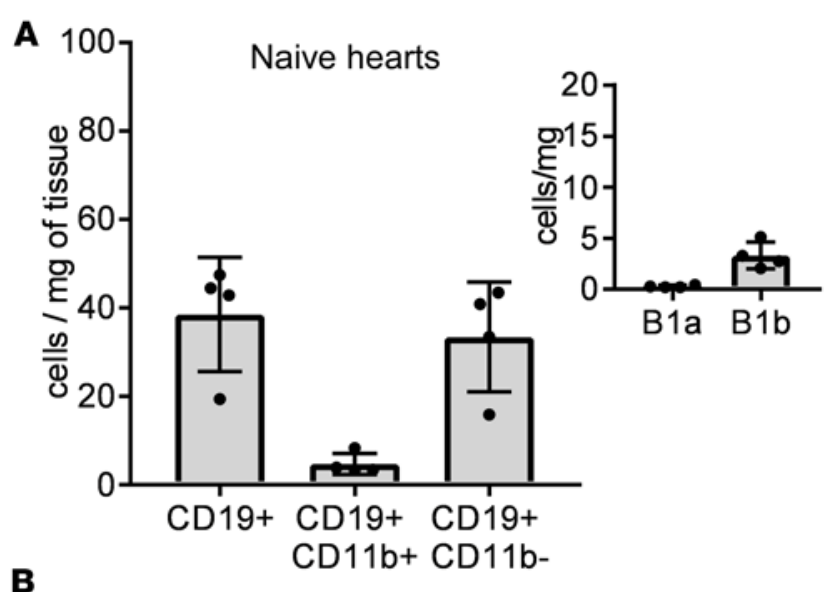

Figure 5. Characterization of subsets of myocardial B lymphocytes at baseline and after DT-induced injury and I/R injury. (A) Analysis of subsets of myocardial CD19+ B lymphocytes in naive hearts ( $n$ =4). (B) Mice expressing the diphtheria toxin receptor (DTR) in the myocardium were exposed to diphtheria toxin (DT) and fed either regular chow (control, gray bars) or chow enriched with pirfenidone (PFD, white bars). Mice were sacrificed at day 4 after DT injection and the heart was collected for analysis of myocardial CD19+ B lymphocytes via flow cytometry $(n=4$ control, $n=3$ pirfenidone). (C) Wild-type mice were subjected to 90 minutes closedchest ischemia followed by reperfusion (I/R injury). Mice were fed either regular chow (control, gray bars) or chow enriched

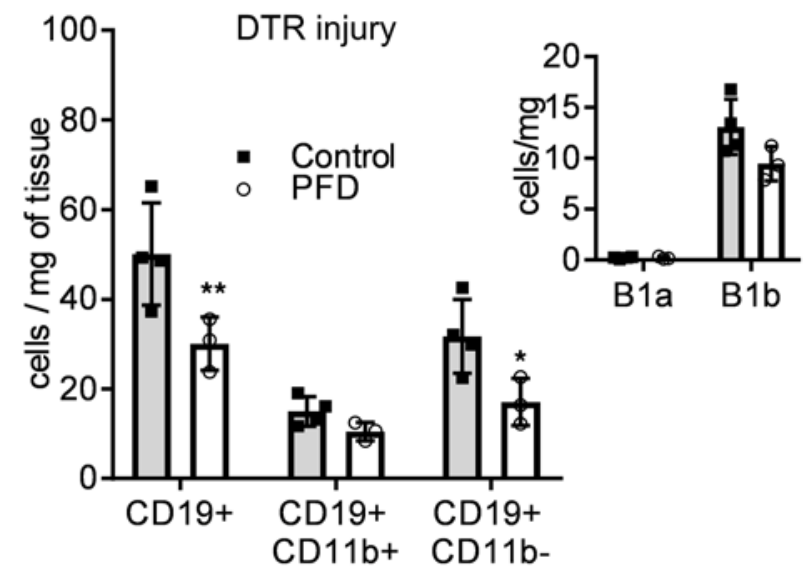
with pirfenidone (PFD, white bars). Mice were sacrificed at day 4 after I/R injury and the heart was collected for analysis of myocardial CD19+ $B$ lymphocytes via flow cytometry ( $n=8$ controls, $n=5$ pirfenidone). ${ }^{*} P<0.05,{ }^{* *} P<0.01,{ }^{* *} P$ $<0.001$ versus control; ${ }^{\dagger} P<0.001$ versus naive hearts. Bars represent the mean, and error bars represent standard deviation. $P$ values were calculated with 2-way ANOVA followed by Tukey's test for multiple comparisons.

C

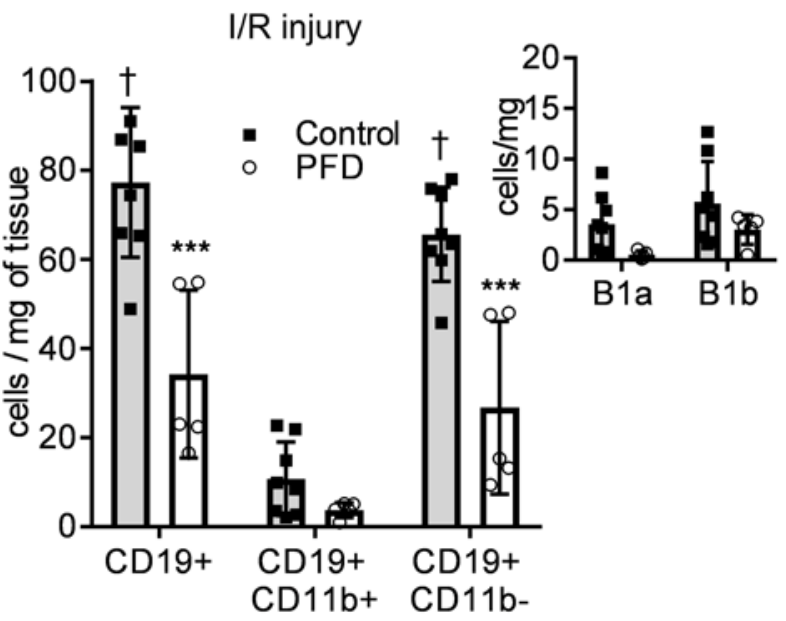

the number of cardiac B cells began to recover on day 4 after I/R injury (i.e., day 12 after injection). However, the total number of $\mathrm{B}$ cells remained less than $10 \%$ that of untreated controls (Supplemental Figure 5C). Notably, while B cell depletion after I/ $\mathrm{R}$ injury significantly reduced $(P<0.01)$ the number of $\mathrm{CD}_{1} 9^{+} \mathrm{CD} 11 \mathrm{~b}^{-}$cells, it did not significantly $(P=0.2)$ reduce the total number of $\mathrm{CD} 19^{+} \mathrm{CD} 11 \mathrm{~b}^{+}$cells (Supplemental Figure 5C). However, when we examined $\mathrm{CD} 19^{+} \mathrm{CD} 11 \mathrm{~b}^{+}$ subsets, treatment with the anti-CD20 antibody did significantly $(P=0.05)$ reduce the number of $\mathrm{B} 1 \mathrm{~b}$ lymphocytes, whereas it had no effect on the number of cardiac B1a lymphocytes $(P=0.58$, Supplemental Figure 5C). There was no significant difference in the heart weight-to-tibia length ratio $(P=0.75$, Figure $6, \mathrm{~B}$ and C), LV mass $(P=0.21$, Figure $6 \mathrm{D}), \mathrm{LV}$ end-diastolic volume $(P=0.40$, Figure $6 \mathrm{E}), \mathrm{LVEF}(P=0.15$, Figure $6 \mathrm{~F})$, or percentage of trichrome-positive myocardium $(P=0.43$, Figure $6, \mathrm{G}$ and $\mathrm{H})$ between the $\mathrm{B}$ cell-depleted mice and B cell-depleted + pirfenidone-treated mice 2 weeks after I/R injury. Thus, the cardioprotective effects of pirfenidone following I/R injury were B cell dependent.

Pirfenidone modulates $B$ cell activation through a TIRAP-dependent signaling pathway. The observation that $B$ cell depletion abrogated the salutary effects of pirfenidone suggested that the beneficial effects of pirfenidone were not necessarily mediated by decreasing the number of B cells following tissue injury, and raised the intriguing possibility that pirfenidone might be modulating the response of $\mathrm{B}$ cells to tissue injury. To gain insight into the mechanism(s) responsible for the immunomodulatory effects of pirfeni- 
done, we performed transcriptional profiling on $\mathrm{CD} 19^{+} \mathrm{CD} 11 \mathrm{~b}^{+}$and $\mathrm{CD} 19^{+} \mathrm{CD} 11 \mathrm{~b}^{-}$cardiac lymphocytes that were FACS isolated from naive, DT-injured mice fed normal chow, and DT-injured mice treated with pirfenidone chow. As shown in Supplemental Figure 6, principal component analysis of the transcriptome of cardiac B cells suggested that the mRNAs of the CD19+CD11b+ (Supplemental Figure 6A) and $\mathrm{CD}{ }^{+} \mathrm{CD} 11 \mathrm{~b}^{-}$(Supplemental Figure 6B) cells from DT-injured hearts had a profile that was distinct from cells from naive hearts. Importantly, the transcriptional profile of the pirfenidone-treated DT-injured mice clustered between the profile of the naive and the untreated hearts, suggesting that pirfenidone modulated the changes in B lymphocyte gene expression that were provoked by cardiac injury. KEGG pathway analysis of mRNAs differentially expressed greater than 2-fold (FDR $<0.05)$ between DT-injured and naive hearts identified 6 pathways that were enriched in the $\mathrm{CD} 19^{+} \mathrm{CD} 11 \mathrm{~b}^{+}$lymphocytes from the DT-injured hearts (Supplemental Figure 6C): hematopoietic cell lineage $\left(P=1 \times 10^{-5}\right)$, cytokine-cytokine receptor interaction $\left(P=3 \times 10^{-3}\right)$, B cell receptor signaling pathway $\left(P=1 \times 10^{-3}\right)$, cell adhesion molecules $(P=$ $0.04)$, antigen processing and presentation $(P=0.04)$, and MAPK signaling $(P=0.05)$. These pathways were not activated in the $\mathrm{CD} 19^{+} \mathrm{CD} 11 \mathrm{~b}^{+}$lymphocytes isolated from the $\mathrm{DT}$ receptor $(\mathrm{DTR})+$ pirfenidone hearts (Supplemental Figure 6C), demonstrating that pirfenidone alters the gene expression changes in $\mathrm{B}$ cells that are driven by cardiac injury. As shown in Supplemental Figure 6D, the KEGG pathway analysis identified 5 pathways in $\mathrm{CD} 19^{+} \mathrm{CD} 11 \mathrm{~b}^{-}$cells: 2 pathways that were also activated in the $\mathrm{CD} 19^{+} \mathrm{CD} 11 \mathrm{~b}^{+}$, specifically hematopoietic cell lineage $\left(P=2 \times 10^{-3}\right)$ and cytokine-cytokine receptor interaction $(P=1 \times$ $\left.10^{-3}\right)$; as well as 3 unique pathways, specifically Toll-like receptor (TLR) signaling $\left(P=1 \times 10^{-3}\right)$, TNF signaling pathway $\left(P=7 \times 10^{-3}\right)$, and chemokine receptor signaling pathway $(P=0.02)$. With the exception of the hematopoietic cell lineage pathway, none of these pathways was significantly enriched in the

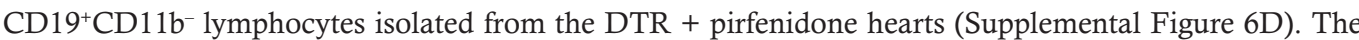
gene lists for each of these KEGG pathways are provided in Supplemental Tables 1 and 2. Viewed together, these studies suggest that myocardial $\mathrm{CD} 19^{+} \mathrm{CD} 11 \mathrm{~b}^{+}$and $\mathrm{CD} 19^{+} \mathrm{CD} 11 \mathrm{~b}^{-}$lymphocytes share common biological responses, but also have unique biological responses to tissue injury, and that both of these common and unique responses to cardiac injury are modulated by pirfenidone.

Noting that prior studies have shown that B cells are activated by engagement of TLR by damage-associated molecular patterns (DAMPs) released by necrotic cells (17), and noting that TLR signaling was present in $\mathrm{CD} 19^{+} \mathrm{CD} 11 \mathrm{~b}^{-}$lymphocytes sorted from DT-injured animals but absent in the same cells sorted from DT-injured animals treated with pirfenidone, we focused our in vitro studies on CD19+ ${ }^{+}$cells using LPS (a classic TLR4 agonist and a T-independent antigen), as well as necrotic cardiac cell extracts, which we and others have shown signal through TLR4 (18). Based on a prior study which showed that activation of TLR signaling was a strong inducer of the costimulatory molecule CD86 in cultured B cells (19), we used upregulation of CD86 as a marker of B cell activation. As shown in Figure 7A, stimulation of peritoneum-derived inflammatory cells (PDICs) with LPS or cytosolic extracts from necrotic cells provoked a robust $(P<$ 0.001 for both) increase in the $\mathrm{CD} 19^{+} \mathrm{CD} 86^{\text {hi }}$ lymphocytes. Importantly, the number of $\mathrm{CD} 19^{+} \mathrm{CD} 86^{\mathrm{hi}}$ was significantly attenuated $(P<0.001$ for both) by pirfenidone. To further explore the role of TLR-mediated signaling, we repeated these experiments in PDICs obtained from thioglycolate-stimulated TIRAP $P^{-/-}$mice. As shown in Figure 7B, necrotic cytosolic extracts did not provoke a significant increase in the percentage of $\mathrm{CD} 19^{+} \mathrm{CD} 86^{\mathrm{hi}}$ lymphocytes, whereas LPS stimulation resulted in a significant increase in the percentage of $\mathrm{CD} 19^{+} \mathrm{CD} 86^{\text {hi }}$ lymphocytes $(P<0.001)$, albeit to a lesser extent than was observed in PDICs from $T I R A P^{+/+}$mice. Notably, treatment with pirfenidone resulted in a small but significant $(P<0.001)$ decrease in $\mathrm{CD} 19^{+} \mathrm{CD} 86^{\text {hi }}$ lymphocytes in LPS-simulated cultures. These findings suggest that, in the context of a mixed population of inflammatory cells, necrotic cytosolic extracts modulate expression of costimulatory molecules on B cells through a TIRAP-dependent mechanism, whereas LPS modulates expression of costimulatory molecules on $\mathrm{B}$ cells through a mechanism that is TIRAP independent. Importantly, both mechanisms were sensitive to treatment with pirfenidone (Figure 7, A and B).

We next sought to determine whether the response of B cells to necrotic cytosolic extracts and LPS were cell autonomous, by repeating the above in vitro experiments in purified B lymphocytes harvested from the spleen. Interestingly, while the effect of LPS on purified B cells was very similar to that observed on PDICs, the effect of necrotic cytosolic extracts was greatly attenuated. As shown in Figure 7C, LPS provoked a significant $(P<0.001)$ increase in $\mathrm{CD} 19^{+} \mathrm{CD} 86^{\mathrm{hi}}$ cells that was partially sensitive to inhibition with pirfenidone. In contrast, necrotic cytosolic extracts induced a small, non-statistically significant $(P=$ 0.62) upregulation of $\mathrm{CD} 19^{+} \mathrm{CD} 86^{\mathrm{hi}} \mathrm{B}$ cells when compared with diluent-treated cells (Figure 7C). Similar 
A

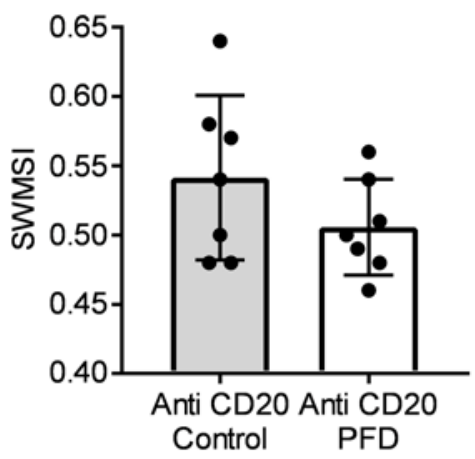

B
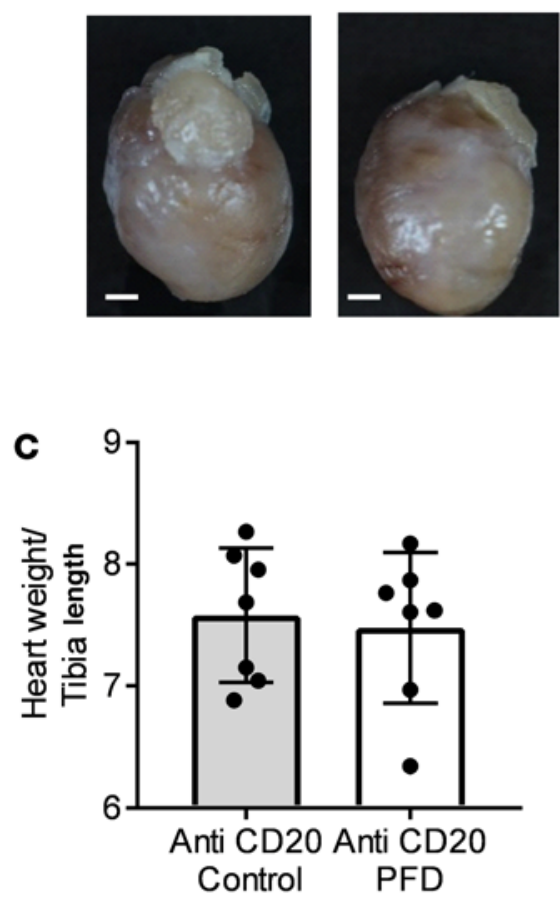

D

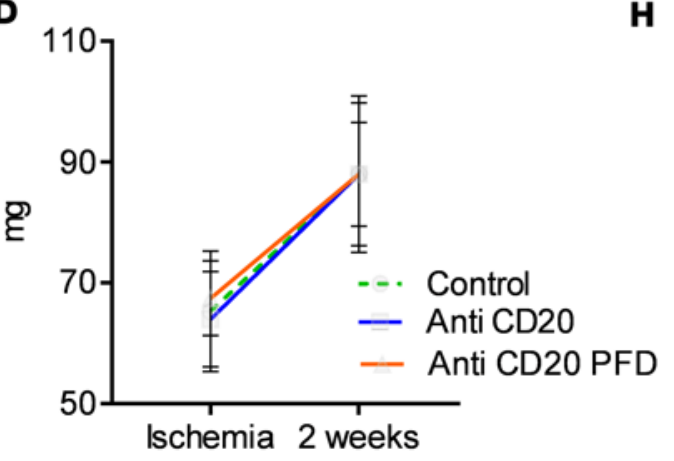

F

G
E
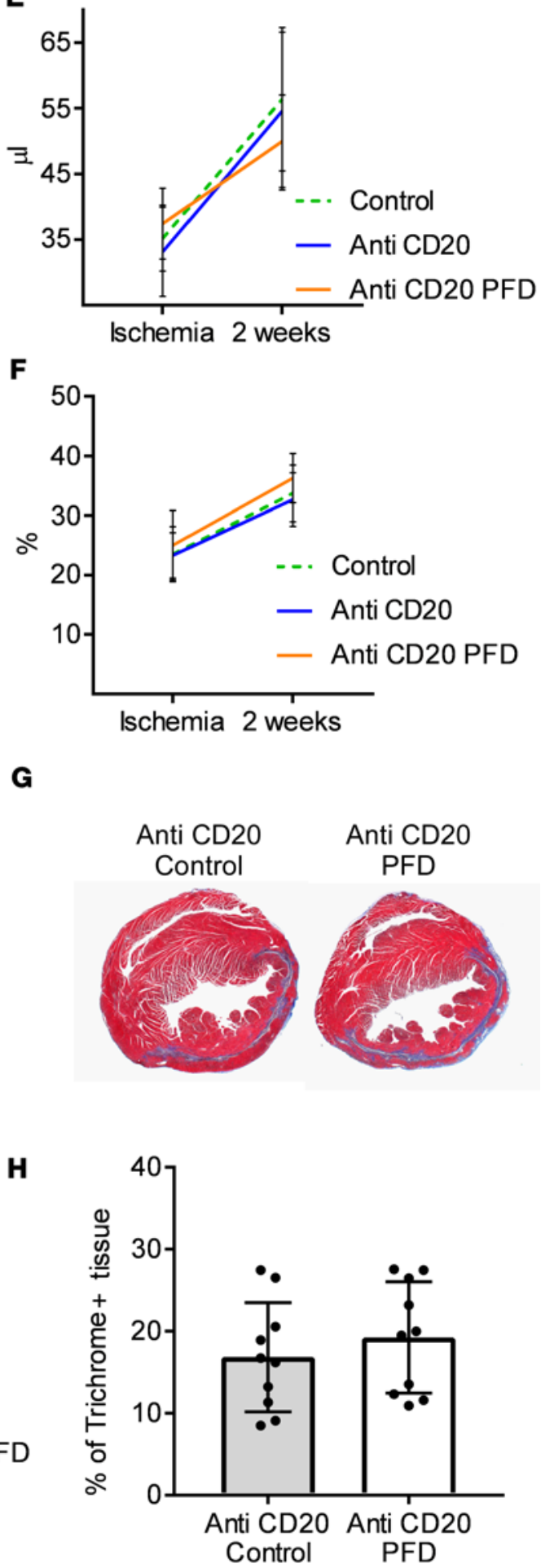

Figure 6. Effect of B cell depletion on pirfenidone cardioprotective effect after I/R injury. Wild-type mice were $B$ cell depleted via injection of anti-CD20 antibody. Seven days after injection of anti-CD2O antibody, B celldepleted mice were subjected to 90 minutes of closed-chest ischemia followed by reperfusion (I/R injury). Mice were fed either chow enriched with pirfenidone (anti-CD20 PFD) or regular chow (anti-CD20 control). (A) Area at risk during closed chest-ischemia as determined by the simplified segmental wall motion score index (SWMSI) at time of ischemia. (B) Representative pictures of hearts harvested from anti-CD20-treated mice (left) and anti-CD20 + pirfenidone-treated animals (right). Scale bar: $1 \mathrm{~mm}$. (C) Gravimetric analysis of hearts harvested from pirfenidone-treated animals (anti-CD2O PFD) and untreated controls (anti-CD20 control), $n=7 /$ group. (D-F) Echocardiographic assessment of myocardial function at the time of ischemia and 2 weeks after I/R injury, $n=7$ / group. Data from non-antiCD20-treated animals already reported in Figure 3, D-F are shown again for comparison only (control). (D) Left ventricular (LV) mass (LVM) by 2-D echocardiography. (E) LV end-diastolic volume (LVEDV). (F) LV ejection fraction (LVEF). (C) Representative trichrome staining of histological sections of hearts from control animals (left panel) and pirfenidone-treated animals (right panel). Original magnification, $\times 1.25$. (H) Quantitative assessment of the percentage of trichrome-positive staining, 2 sections analyzed per heart, $n=10$ sections/group. Bars represent the mean, and error bars represent standard deviation. $P$ values were calculated with Student's $t$ test. 
results were obtained in splenic B lymphocytes from TIRAP $P^{-1-}$ animals (Figure 7D). Viewed together, these findings suggest that the B cell response to LPS and its modulation by pirfenidone are cell autonomous, and are mediated, at least in part, through TIRAP-dependent signaling. In contrast, the response of B cells to necrotic cell extracts (NCEs) and the immunomodulatory effects of pirfenidone are non-cell autonomous, which suggest that they are likely to be context and tissue dependent.

To determine whether our findings with respect to activation of $\mathrm{CD} 19^{+} \mathrm{B}$ cells in vitro were relevant in vivo, we measured the number of myocardial $\mathrm{CD} 19^{+} \mathrm{CD} 86^{\mathrm{hi}} \mathrm{B}$ cells isolated from the hearts of mice from the DT-induced injury model and the closed-chest I/R model in the presence and absence of pirfenidone. As shown in Figure 7E, treatment with pirfenidone was associated with a significant $(P<0.04)$ decrease in the number of myocardial $\mathrm{CD} 19^{+} \mathrm{CD} 86^{\mathrm{hi}} \mathrm{B}$ lymphocytes in the DT-induced injury model and Figure $7 \mathrm{~F}$ shows that treatment with pirfenidone was associated with a significant $(P<0.03)$ decrease in the number of myocardial $\mathrm{CD} 19^{+} \mathrm{CD} 86^{\text {hi }} \mathrm{B}$ cells following I/R injury.

\section{Discussion}

In this study, we investigated the cardioprotective effects of pirfenidone, a small molecule that is FDA approved for the treatment of idiopathic pulmonary fibrosis, and provide evidence that pirfenidone exerts beneficial effects in the heart through a potentially unique mechanism that involves immune modulation of the cardiac B lymphocyte response to tissue injury. The following lines of evidence support this statement. First, treatment with pirfenidone resulted in improved survival in a genetic model of cardiac myocyte injury. Notably, the improved survival was not secondary to a decrease in apoptotic or necrotic cardiac myocyte cell death (Figure 1). Moreover, the improved survival was not secondary to decreased influx of Ly6G $\mathrm{G}^{+}$ neutrophils, Ly6C ${ }^{+} \mathrm{CD} 64^{10 /-}$ monocytes, or $\mathrm{CD} 64^{+} \mathrm{Ly} 6 \mathrm{C}^{\mathrm{lo} /-}$ macrophages. Rather, we observed a significant decrease in the number of $\mathrm{CD}_{1} 9^{+}$lymphocytes in the heart following pirfenidone treatment (Figure 2). Second, treatment with pirfenidone significantly reduced cardiac remodeling after closed-chest I/R injury, as demonstrated by a decrease in the heart weight-to-tibia length ratio, LV mass, and LV end diastolic volume (Figure 3). Importantly, the salutary effects of pirfenidone were present whether animals were pretreated with the drug or it was administered after I/R injury (Supplemental Figure 2). The attenuation in LV remodeling was not secondary to decreased influx of $\mathrm{Ly} 6 \mathrm{G}^{+}$neutrophils, $\mathrm{Ly} 6 \mathrm{C}^{+} \mathrm{CD} 64^{\mathrm{lo} /-}$, monocytes, or $\mathrm{CD}^{+} 4^{+} \mathrm{Ly} 6 \mathrm{C}^{\mathrm{lo} /-}$ macrophages (Figure 4). Similar to the observations made in the DT-induced injury model, we observed a significant decrease in $\mathrm{CD} 19^{+}$lymphocytes in the heart following pirfenidone treatment (Figure 4). Remarkably, B cell depletion with anti-CD20 antibody attenuated the cardioprotective effects of pirfenidone (Figure 6), suggesting that although the effects of pirfenidone were B cell dependent, they were not necessarily mediated by decreasing the number of B cells in the injured heart. Third, treatment with pirfenidone altered the biological response to tissue injury of myocardial $\mathrm{CD} 19^{+} \mathrm{CD} 11 \mathrm{~b}^{+}$lymphocytes and of a population of $\mathrm{CD} 19^{+} \mathrm{CD} 11 \mathrm{~b}^{-} \mathrm{CD} 23^{-} \mathrm{CD} 21^{-} \mathrm{IgM}^{\mathrm{lo}} \mathrm{IgD} \mathrm{D}^{\text {hi }}$ lymphocytes in vivo (Supplemental Figure 6), as well as decreased the expression CD86 on B cells in vitro and in vivo (Figure 7). While our data do not exclude an effect of pirfenidone on other cell types, viewed together the above observations suggest that pirfenidone is cardioprotective through a unique mechanism that involves immune modulation of myocardial B cell subsets. Although we did not observe an antifibrotic effect of pirfenidone after I/R injury, as has been reported previously by some $(4,5,7,9-12)$, but not all groups (8), this discrepancy may be secondary to differences in the experimental models used and the duration and/or doses of pirfenidone that were used.

Myocardial B lymphocytes in health and disease. Recent studies have shown that several discrete populations of leukocytes reside in the normal adult murine myocardium. Antigen-presenting mononuclear cells $\left(\mathrm{CD} 11 \mathrm{~b}^{+} \mathrm{CD} 11 \mathrm{c}^{+} \mathrm{F} 4 / 80^{+}\right.$and $\left.\mathrm{MHCII}^{+}\right)$represent the most prominent population, followed by B cells, monocytes, and $\mathrm{T}$ cells (20). Although there has been tremendous recent interest in the role of macrophages, dendritic cells, and $\mathrm{T}$ cells in the heart, comparatively less is known about the role of B cells. Very little is known about the composition of myocardial B cells in naive hearts. Ramos et al. have shown that the naive adult murine myocardium harbors 2 populations of $\mathrm{B} 220^{+}(\mathrm{CD} 45 \mathrm{R})$ lymphocytes, a larger population of $\mathrm{IgM}^{\mathrm{hi}} \mathrm{Ig}_{-}$ $\mathrm{D}^{10}$ cells, and a smaller population of $\operatorname{IgM}^{\mathrm{lo}} \operatorname{IgD}{ }^{\text {hi }}$ cells (21); however, neither the ontology nor functional role of these 2 populations of lymphocytes was characterized in this study. The existing literature suggests that B lymphocytes play an important role in chronic LV remodeling following cardiac injury, although the specific B cell-mediated mechanisms have not been identified completely. Three different groups have studied the role of myocardial B lymphocytes following acute coronary ligation (MI). Both Yan et al. (22) and Zouggari et al. (23) reported that the number of myocardial $\mathrm{CD} 19^{+}$cells increased after MI. Zouggari showed that 
A

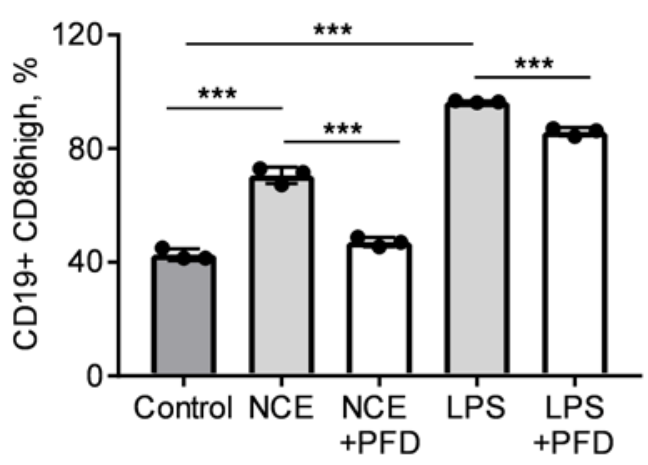

C

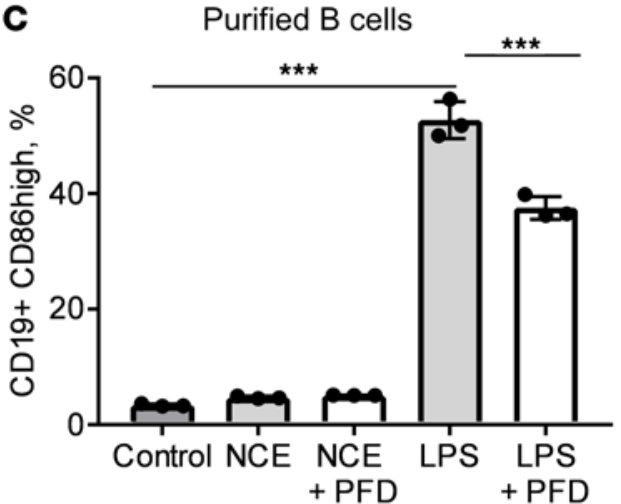

$\mathbf{E}$

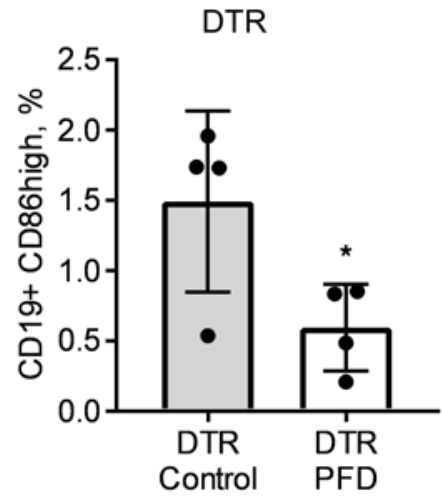

B

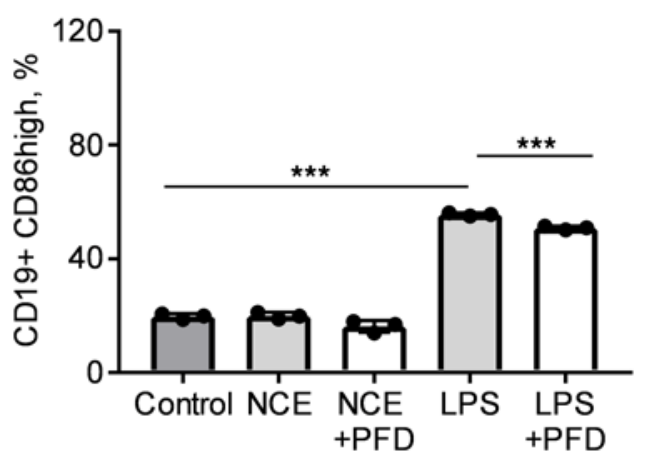

D

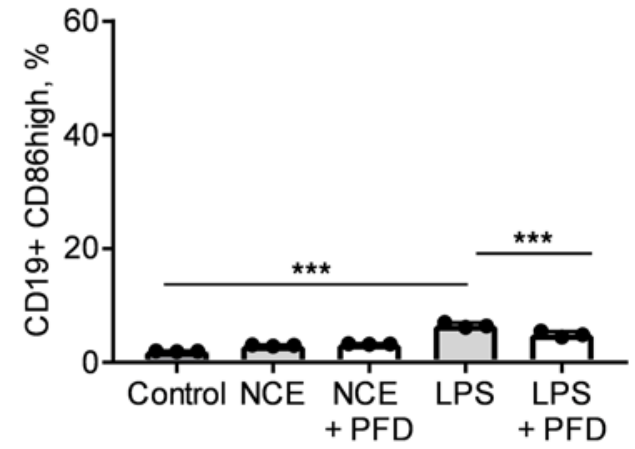

$\mathbf{F}$

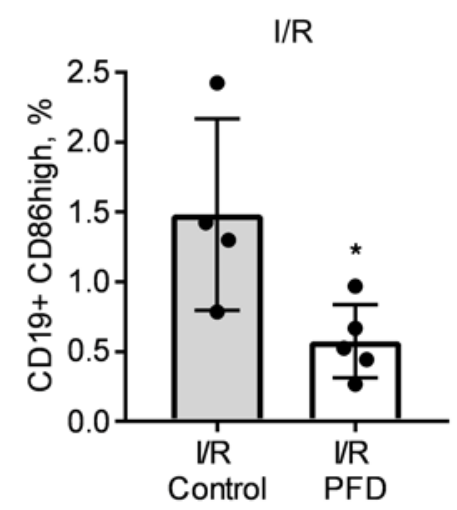

Figure 7. Analysis of B cell activation in wild-type and TIRAP-deficient immune cells. (A and B) Unfractionated peritoneum-derived inflammatory cells (PDICs) were collected on day 4 after intraperitoneal injection of thioglycollate and placed in culture. Cells were cultured in media alone (control), in the presence of necrotic cell extracts from H9c2 cells (NCES), in the presence of NCEs and pirfenidone (NCE-PFD), in the presence of LPS (LPS), or in the presence of LPS and pirfenidone (LPS-PFD). After 24 hours of culture, cells were collected for flow cytometric analysis and the prevalence of $\mathrm{CD}{ }^{+} \mathrm{CD}^{\mathrm{h}} \mathrm{h}^{\mathrm{hi}}$ cells was quantified. Three biological replicates per experiment are reported. (A) Results from inflammatory cells collected from wild-type mice. (B) Results from inflammatory cells collected from TIRAP ${ }^{-1-}$ animals. (C and D) B lymphocytes were purified from the spleen and cultured for 24 hours under the same conditions described for panels A and B. After 24 hours of culture, cells were collected for flow cytometric analysis and the prevalence of CD19+CD86 ${ }^{\text {hi }}$ cells was quantified. Three biological replicates per experiment are reported. Panel $\mathbf{C}$ shows results from splenic B cells purified from wild-type mice, and panel $\mathbf{D}$ shows results from B lymphocytes collected from TIRAP ${ }^{-/-}$animals. (E and F) Mice were subjected to acute myocardial injury either by exposure to diphtheria toxin (DT) (E) or through 90 minutes of closed-chest ischemia followed by reperfusion (I/R) (F). Mice were fed regular chow (control) or chow enriched with pirfenidone (PFD). On day 4 after injury, the myocardium was collected and analyzed via flow cytometry to assess the number of myocardial CD19+CD86 hi cells. In E, $n=4$ per/group; in F, $n=4$ control, $n=5$ PFD. ${ }^{*} P<0.05$, ${ }^{* *} P<0.001$. Bars represent the mean, and error bars represent standard deviation. $P$ values were calculated with 1-way ANOVA followed by Tukey's test for multiple comparisons in panels $\mathbf{A}-\mathbf{D}$ and with Student's $t$ test in panels $\mathbf{E}$ and $\mathbf{F}$. 
$\mathrm{CD}_{19}{ }^{+} \mathrm{IgD}^{+} \mathrm{IgM}^{\text {lo }} \mathrm{B}$ lymphocytes influx into the infarcted myocardium and contribute to adverse LV remodeling by recruiting Ly $6 \mathrm{C}^{+}$monocytes from the bone marrow through a CCL7-dependent mechanism (23). In contrast, a recent study found that $\mathrm{CD} 19^{+}$cells are recruited preferentially to fat-associated lymphoid clusters in pericardial adipose tissue, with minimal detection of $\mathrm{CD}_{19}{ }^{+}$cells in the myocardium after MI (24). Using a nonsurgical model of cardiac injury, Cordero-Reyes et al. showed that adverse cardiac remodeling was attenuated by antibody-dependent depletion of B cells in WT mice (25). Although the mechanisms of action of B lymphocyte-mediated myocardial damage have not been fully elucidated, there are several mechanisms that have been proposed, including dysregulation of B cell subpopulations (B1 vs. B2 vs. regulatory B cells), deposition of IgM or IgG antibodies, as well as B cell-mediated recruitment of Ly6C ${ }^{+}$monocytes (23). In this regard, it is interesting to note that Zhang et al. showed that natural IgMs play a role in myocardial I/R injury, although they did not specifically study myocardial B lymphocytes (26).

The results of the present study both confirm and expand on prior studies that have described myocardial B lymphocytes and suggested that B lymphocytes play an important role in modulating adverse cardiac remodeling following cardiac injury. Here we show for the first time to our knowledge that in naive murine hearts the majority of $\mathrm{B}$ lymphocytes are $\mathrm{CD} 19^{+} \mathrm{CD} 11 \mathrm{~b}^{-} \mathrm{CD} 21^{-} \mathrm{CD} 23^{-} \mathrm{CD} 11 \mathrm{c}^{-} \mathrm{IgD}^{+} \mathrm{IgM}^{\mathrm{lo} /-}$, whereas a minority of myocardial B lymphocytes are $\mathrm{CD} 19^{+} \mathrm{CD} 11 \mathrm{~b}^{+}$. In this latter group, a smaller fraction express $\mathrm{CD} 5$, which is characteristic of B1a lymphocytes, and a larger fraction is $\mathrm{CD}^{-}$, which is characteristic of B1b lymphocytes (16). Although the phenotype of the $\mathrm{CD} 19^{+} \mathrm{CD} 11 \mathrm{~b}^{-}$cells that we describe overlaps the phenotypic description of the $\mathrm{B}$ cells that accumulated in the myocardium after infarction in the report by Zouggari et al. (i.e., $\mathrm{CD}^{1} 9^{+} \operatorname{IgD}^{+} \operatorname{IgM}^{10}$ ), it bears emphasis that the precise ontogeny of the $\mathrm{CD} 19^{+} \mathrm{CD} 11 \mathrm{~b}$ $\mathrm{CD}_{21}{ }^{-} \mathrm{CD} 23^{-} \mathrm{CD} 11 \mathrm{c}^{-} \mathrm{IgD}^{+} \mathrm{IgM}^{\mathrm{lo} /-}$ cells described herein is not known. To our knowledge, the phenotype of these cells does not match the profile of any previously described B cell subset (27). B cells can be broadly characterized into B1 and B2 lymphocytes, which are classically considered innate and adaptive, respectively. Whereas B1a lymphocyte development occurs primarily during fetal and perinatal life, B1b and B2 lymphocyte production continues throughout adult life. The observation that these cells express relatively high levels of $\mathrm{IgD}$, which is characteristic of $\mathrm{B} 2$ cells, and low levels of $\operatorname{IgM}$, which are characteristically expressed by B1 cells, suggests that these cells are not B1 cells (23). Of note, several authors have reported that a distinct population of mature $\mathrm{CD} 19^{+} \mathrm{CD} 21^{-} \mathrm{CD} 23^{-} \mathrm{B}$ cells accumulate in aging mice (28). However, these age-associated $\mathrm{B}$ cells are $\mathrm{CD} 11 \mathrm{c}^{+}$and express the transcription factor $\mathrm{T}$-bet, whereas we did not detect CD11c expression via FACS, nor gene expression of T-bet by RNA sequencing (data not shown). Our results further expand on the existing literature on myocardial B cells also by showing that unique subsets of B lymphocytes increase in the heart following I/R injury (Figure 5), and that different subsets of myocardial B lymphocytes $\left(\mathrm{CD} 19^{+} \mathrm{CD} 11 \mathrm{~b}^{+}\right.$and $\mathrm{CD} 19^{+} \mathrm{CD} 11 \mathrm{~b}^{-}$lymphocytes) are activated differentially following cardiac injury, in a process that is modulated by pirfenidone (Supplemental Figure 6). Although we did not observe a statistically significant increase in CD19+ lymphocytes in the heart after DT-mediated cardiac injury, the overall trends were similar to those observed with I/R injury. The quantitative differences in B cell response may be secondary to differences in the degree of tissue injury and/or differences in B lymphocyte kinetics in the 2 different injury models.

Our findings suggest that B cells are required for the cardioprotective effects of pirfenidone after acute tissue injury and that B cell depletion does not mimic the cardioprotective effects of pirfenidone following tissue injury in vivo. Although these results are seemingly incongruent at first blush, they suggest a more nuanced model supported by our transcriptional profiling studies in vivo (Supplemental Figure 6), wherein pirfenidone exerts its beneficial effects by modulating the immune response of B cells to tissue injury. Based on the results of our informatics analysis we hypothesized that activation of TLR signaling in response to the release of the cytosolic contents from necrotic cells (i.e., DAMPS) might activate B cells through a pirfenidone-sensitive process. We found that NCEs were sufficient to upregulate the costimulatory molecule CD86 in B lymphocytes isolated from cultures of mixed inflammatory cells, whereas NCEs were not sufficient to upregulate CD86 in purified B cell preparations. In contrast, LPS, a classic TLR4 agonist, induced upregulation of CD86 in $\mathrm{CD} 19^{+}$cells in PDICs as well as in purified B cell preparations. These findings are consistent with prior reports showing that the B lymphocyte response to TLR agonists is context dependent (29), and suggest that B lymphocyte activation in response to NCE is, at least in part, non-cell autonomous. Importantly, we observed that pirfenidone significantly attenuated the number $\mathrm{CD} 19^{+} \mathrm{CD} 86^{+}$lymphocytes in PDIC cultures stimulated with NCEs or LPS. Consistent with our in vitro studies, we observed that there was a significant decrease in $\mathrm{CD} 19^{+} \mathrm{CD} 86^{\mathrm{hi}}$ lymphocytes in the hearts of DT-injured and I/R-injured mice treated with pirfenidone when 
compared with untreated controls (Figure 7, E and F). Our in vitro results with $T I R A P^{-1-}$ cells (Figure 7) suggest that activation of $\mathrm{B}$ cells by NCEs is TIRAP dependent. However, LPS-induced activation of $\mathrm{B}$ cells in vitro was attenuated but not abrogated in $T I R A P^{-/-}$mice. This is consistent with prior observations that LPS signaling can activate multiple intracellular pathways in B cells (30).

Our work has some important limitations. First, we did not specifically compare the effect of pirfenidone and anti-CD20 treatment. Second, we did not measure the effect of pirfenidone treatment in IgG-treated animals. In addition, we did not determine which actions of B cells accentuate adverse remodeling following acute myocardial injury. Further work will be needed to detail the cell-autonomous versus non-cell-autonomous role of TIRAP during B cell activation in the context of the acute inflammatory response to sterile injury, to understand if pirfenidone-mediated modulation of $B$ cells is dependent on the modulation of other signaling pathways, including TGF- $\beta$ signaling, and to define the mechanistic basis of B cell contribution to adverse cardiac remodeling.

Although the scope of these studies was not intended to define the molecular mechanisms of pirfenidone, our findings are consistent with prior reports that have shown that pirfenidone inhibits TLR signaling through the MAPK signaling cascade (31), as well as studies that show that pirfenidone inhibits MAPK signaling in the heart $(10,12)$. Importantly, this is the first study to our knowledge that demonstrates a direct cellular target that mediates the beneficial effects of pirfenidone in vivo.

In our work we found that pirfenidone-dependent immunomodulation of the cardiac inflammatory infiltrate improved cardiac function after acute injury. Surprisingly, treatment with pirfenidone was not associated with a reduction in troponin on day 4 after administration of DT (Figure 1B) nor a reduction in infarct size after I/R injury, as assessed by trichrome staining (Figure 3, G and $\mathrm{H}$ ). This finding suggests that cardiac B lymphocytes might modulate cardiac remodeling after injury. Further work will be needed to understand the significance of this intriguing observation.

Conclusions. Although it has been long recognized that the innate immune system plays an important role in the adverse cardiac remodeling and myocardial dysfunction that ensues following acute myocardial injury, the quest to identify viable therapeutic targets has been illusory to date, because of the inherent duality of the innate immune response, which is required for initiating cardiac repair following tissue injury (32). Here we show a previously unappreciated complexity of the B lymphocyte component of the acute inflammatory response triggered by acute myocardial damage, and we show that B cells' response to myocardial injury can be modulated through a small-molecule-based approach, as opposed to an antibody-based approach. This raises the interesting question of whether pirfenidone might be repurposed as an immunomodulatory agent in the setting of I/R injury following percutaneous cardiac angioplasty for ST-segment myocardial infarction. In contrast to monocytes/macrophages, which are essential for cardiac repair, B lymphocytes function, at least in part, by modulating interactions between T cells, cardiac macrophages, and dendritic cells. Accordingly, immunomodulatory strategies that are designed to target B cells may be less likely to lead to untoward effects, than are strategies that are aimed at cytokines and cell types that are critical for myocardial repair. Lastly, given the increasing recognition of the importance of B lymphocytes in the heart (23-25), these studies may also serve the heuristic purpose of focusing future studies on better understanding the origins, dynamics, temporal development, and functional significance of the myocardial B lymphocytes in health, disease, and aging.

\section{Methods}

\section{Mouse injury models}

To evaluate the effects of pirfenidone on acute myocardial injury, we used 2 distinct in vivo experimental models of myocardial injury: a nonsurgical model of selective cardiac myocyte cell death (13) and a well-characterized surgical model of closed-chest I/R injury $(15,33)$. The mice for these experiments were bred and maintained at the Washington University School of Medicine and all experimental procedures were done in accordance with the animal use oversight committee.

DT-induced injury model. Mlc2v-Cre mice (C57BL/6J, Jackson Laboratories) were crossed to Rosa26-DT mice (C57BL/6J) to generate lines of Rosa26-DT ${ }^{\text {Mlc2v-Cre }}$ mice. Female mice, 9-40 weeks of age, were injected i.p. with $2.5 \mathrm{ng} / \mathrm{g}$ of DT (Sigma-Aldrich). DT was solubilized according to the manufacturer's instructions and stored in aliquots at a concentration of $2,000 \mathrm{ng} / \mu 1$ at $-80^{\circ} \mathrm{C}$. Aliquots stored at $-80^{\circ} \mathrm{C}$ were diluted to $20 \mathrm{ng} /$ $\mu \mathrm{l}$ in PBS $1-10$ days prior to use and stored at $-20^{\circ} \mathrm{C}$. DT was diluted to a final concentration of $1 \mathrm{ng} / \mu \mathrm{l}$ in PBS 
immediately prior to injections. Littermate controls were used for each experiment. Mice were treated and studied as they became available and the results from all available experiments were compiled for analyses.

To assess the extent of myocyte injury following DT-induced injury, we measured troponin release and Evan's blue dye uptake. Troponin was measured day 4 after injection of DT at the time of terminal sacrifice. Blood was collected in BD Microtainer tubes and the serum was diluted 1:4 in PBS (50 $\mu 1$ serum +150 $\mu 1$ PBS). Serum troponin was measured using a commercial chemiluminescent microparticle assay (Abbot Laboratories). Evan's blue dye uptake was assessed on day 4 after DT injection, as described previously $(34,35)$. Hearts were examined at the level of the papillary muscle using ImageJ software (NIH). Data are expressed as the percentage area of the myocardium with red fluorescence.

$I / R$ injury. Hearts from WT mice (C57BL/6J) were subjected to I/R injury using the closed-chest I/R model developed by Entman and colleagues (33), and modified as we have described previously (15). For the studies described herein we used 9- to 10-week-old mice that were instrumented, then at 1 week after instrumentation were anesthetized with $1.5 \%$ isoflurane, and subjected to 90 minutes of closed-chest ischemia, followed by reperfusion for 2 weeks.

\section{Pirfenidone treatment}

In order to administer pirfenidone, mice were fed powdered chow mixed with pirfenidone (eNovation Chemicals, catalog D404655) at $0.5 \%$ by weight or powdered chow alone. This dosage has been commonly used in rodent models and has been suggested to result in plasma concentrations similar to those observed in humans treated with pirfenidone (36). Unless specifically noted, mice were switched to a diet enriched with pirfenidone 3 days prior to DT-induced injury or I/R injury, and maintained on this diet throughout the duration of the experiments. In parallel studies, we fed mice normal chow prior to I/R injury and then administered pirfenidone acutely at the time of cardiac injury. For these latter studies, mice were injected i.p. with diluent (200 $\mu \mathrm{l}$ PBS) or i.p. with pirfenidone (200 $\mu$ l of pirfenidone, $5 \mathrm{mg} / \mathrm{ml}$ in PBS) after reperfusion and the morning after I/R injury (2 injections total). They were switched to pirfenidone-enriched diet or control diet the evening of I/R injury and maintained on the same diet until completion of the experiment.

\section{$B$ cell depletion}

For the B cell depletion studies, mice were injected with $100 \mu \mathrm{g}$ of anti-CD20 antibody (Biolegend, clone SA271G2, catalog 152104) through the jugular vein. The injected antibody was diluted in PBS, $\mathrm{pH} 7.2$, containing no preservative (endotoxin level $<0.01 \mathrm{U} / \mu \mathrm{g}$ of protein) and filtered using a $0.2-\mu \mathrm{m}$ filter. Immunodepletion was assessed at different time points, as described in the results section.

\section{Gravimetric and histological analysis}

Rosa26-DT ${ }^{\text {Mc2v-Cre }}$ and littermate control WT mice were euthanized 14 days after DT injection, and the hearts were removed and weighed to determine the heart weight/tibia length ratio. WT mice were euthanized 14 days after I/R injury and the hearts were removed and weighed to determine the heart weight/tibia length ratio. Hearts were processed, paraffin-embedded, and stained with hematoxylin and eosin and Masson's trichrome, as described previously (37). TUNEL staining was performed with the Millipore S7200 ApopTag Peroxidase In Situ Oligo Ligation Apoptosis Detection Kit according to the manufacturer's instructions.

\section{Echocardiographic studies}

Image acquisition. Ultrasound examination of the cardiovascular system was performed using a Vevo 2100 Ultrasound System (VisualSonics Inc.) equipped with a 30-MHz linear-array transducer, as previously described (15).

Imaging protocol. Mice were imaged by echocardiography at the time of ischemia and 2 weeks after reperfusion to evaluate LV structure and function, as described previously (38). The echocardiographer was blinded to study group assignment. Animals with poor acoustic windows or small areas at risk $(<0.45)$ at the time of ischemia were excluded from further analysis.

\section{Cell culture}

PDICs were harvested from the peritoneal cavity of thioglycolate-stimulated WT and TIRAP ${ }^{-1-}$ mice. PDIC cultures contain an admixture of inflammatory cells, including CD19+ B cells (39). TIRAP-deficient mice were a gift from Ruslan Medzhitov (Yale University, New Haven, Connecticut, USA). PDICs were harvested on day 4 after i.p. injection of $1 \mathrm{ml}$ of thioglycolate medium (Millipore). Mice were euthanized, and the 
peritoneal cavity was washed 3-4 times with $3 \mathrm{ml}$ of DMEM containing $10 \%$ FCS. A 3-ml syringe with an 18-gauge needle was used to inject the peritoneal cavity and retrieve the medium containing PDICs. Harvested cells were centrifuged at $250 \mathrm{~g}$ for 5 minutes, resuspended in $5 \mathrm{ml}$ of media, and then filtered through a $40-\mu \mathrm{m}$ strainer. Cells were plated at approximately 1.5 million cells $/ \mathrm{ml}$ in 12 - or 24 -well plates. Primary spleen-derived B lymphocytes were isolated with the MagniSort Mouse B cell Enrichment Kit (Invitrogen) according to manufacturer's instructions and plated in 96-well plates at 1 million cells $/ \mathrm{ml}$. The purity of isolated B cells was checked by flow cytometry and was greater than $85 \%$ in all reported experiments. Both PDICs and primary B lymphocytes were cultured in DMEM with 10\% FCS, $1 \mathrm{mM}$ sodium pyruvate, penicillin/ streptomycin (GIBCO, 1×), 2 mM L-glutamine, $10 \mathrm{mM}$ Hepes, and $55 \mu \mathrm{M}$ 2-mercaptoethanol.

We used LPS-EB ultrapure LPS (InvivoGen) or NCEs to stimulate the cell cultures. For stimulation with LPS, LPS was added at $100 \mathrm{ng} / \mathrm{ml}$ at the time of plating. NCEs were prepared from H9c2 cells, in a similar manner to the protocol described for mouse heart and mouse liver extracts (18). For stimulation, NCEs were added at a final concentration of $10 \mu \mathrm{g} / \mathrm{ml}$ at the time the cells were plated. LPS and NCE stimulation was performed in the presence and absence of pirfenidone for 24 hours. Pirfenidone was solubilized $(10 \mathrm{mg} / \mathrm{ml})$ in DMEM by 2 rounds of gentle heating in a water bath at $65^{\circ} \mathrm{C}$ for 5 minutes, followed by vortexing for 20 seconds. The pirfenidone solution was stored at $4^{\circ} \mathrm{C}$ and used within 24 hours of preparation. Pirfenidone was added at a final concentration of $150 \mathrm{ng} / \mathrm{ml}$. For FACS analysis, both adherent and nonadherent cultured cells were collected. Nonadherent cells were collected by pipetting up and down in the culture media prior to media collection and a single rapid wash with Cell Stripper (Corning), and then gently centrifuged at $250 \mathrm{~g}$ for 3 minutes. To collect the adherent fraction, cells were incubated for 30 minutes at $37^{\circ} \mathrm{C}$ with Cell Stripper and then detached by gentle pipetting followed by mechanical detachment with a cell lifter. The adherent cells were mixed with the nonadherent cells, spun down at $250 \mathrm{~g}$ for 3 minutes, and resuspended in $300 \mu \mathrm{l}$ of FACS buffer for further processing.

\section{Flow cytometry}

For flow cytometry experiments, mice were euthanized in $\mathrm{CO}_{2}$ chamber and the hearts were perfused with cold PBS, carefully dissected from extracardiac tissue under a stereo microscope, finely minced, suspended in $3 \mathrm{ml}$ of DMEM, and then digested with $120 \mathrm{U}$ of DNAse (Sigma-Aldrich), $180 \mathrm{U}$ of hyaluronidase (Sigma-Aldrich), and 1,350 U of collagenase (Sigma-Aldrich) for 60 minutes at $37^{\circ} \mathrm{C}$. The digested material was filtered through $40-\mu \mathrm{m}$ filters and pelleted by centrifugation $\left(250 \mathrm{~g}\right.$ for 3 minutes at $\left.4^{\circ} \mathrm{C}\right)$ in HBSS supplemented with $2 \%$ FCS plus $0.2 \%$ bovine serum albumin (BSA). Red blood cells were lysed in ACK lysis buffer (Invitrogen) for 15 minutes on ice and the remaining cells were resuspended in $300 \mu$ of FACS buffer (PBS with 2\% FCS and 2 mM EDTA). Cultured cells were collected for FACS analysis as described above and resuspended in $300 \mu 1$ of FACS buffer. Samples were labeled with fluorescently conjugated antibodies. A list of all antibodies used including brand, clone number, and fluorophores used are provided in Supplemental Table 3. Cells were stained for 45 minutes on ice, and washed in FACS buffer prior to analysis. All antibodies from BioLegend were used at $0.2 \mu 1$ per $300 \mu 1$ sample, while antibodies from BD Biosciences were used at $0.4 \mu 1$ per $300 \mu 1$ sample. FACS was performed on Becton Dickinson analyzers (LSRII, Canto, X20, or Fortessa). Compensation controls were generated using UltraComp eBeads (Invitrogen) and verified on single-color control samples obtained by staining primary splenocytes. Gating strategies are summarized in Supplemental Figures 1, 3, and 4. For flow cytometry experiments that involved the DT injury model, we collected serum via retro-orbital puncture immediately prior to terminal sacrifice for quantification of troponin. In pilot studies we observed that mice with a serum troponin below $1 \mathrm{ng} / \mathrm{ml}$ did not have appreciable tissue injury; therefore, mice with serum troponin below $1 \mathrm{ng} / \mathrm{ml}$ were excluded from further analysis. Cell sorting was performed using a FACSAria instrument (Becton Dickinson) at the Washington University Department of Pathology Flow Cytometry and Sorting Core.

\section{Transcriptional profiling}

We performed transcriptional profiling of cardiac lymphocytes that were isolated from naive and DTR-injured hearts in the presence and absence of pirfenidone. We FACS sorted CD $19^{+} C D 11 b^{+}$and CD $19^{+} C D 11 b^{-}$ cardiac lymphocytes from naive, DT-injured, and DT + pirfenidone hearts directly into lysis buffer for RNA isolation. A total of 600 to 12,000 cells were sorted from each heart. RNA was extracted using the QuickRNA MicroPrep (Zymo Research), according to the manufacturer's instructions. RNA was eluted in $11 \mu \mathrm{l}$ and the entire volume was submitted for whole-transcriptome analysis. Myocardial B lymphocytes 
were sorted from 8 naive animals, 6 DT mice, and 3 DT + pirfenidone-treated mice. Total RNA obtained from sorted B cell populations was selected for polyadenylated RNA and converted to RNA-sequencing libraries using the SMARTer v2 kit from Clontech. Single-end, 50-bp reads were obtained on an Illumina HiSeq 3000 and aligned to the Illumina iGenomes GRCm38_Ensembl release of the mouse transcriptome using Tophat2.1 (40), yielding an average of $1.8 \times 10^{7}$ aligned reads per sample. Gene-level quantification was performed using HTSeq 2 (41). mRNAs were included in downstream analyses if present in all biological replicates in any of the 6 experimental groups $\left(\mathrm{CD} 19^{+} \mathrm{CD} 11 \mathrm{~b}^{+}\right.$and $\mathrm{CD} 19^{+} \mathrm{CD} 11 \mathrm{~b}^{-}$from WT, DT, and $\mathrm{DT}+$ pirfenidone) at an abundance of at least 1 read per million. A total of 12,097 mRNAs were obtained

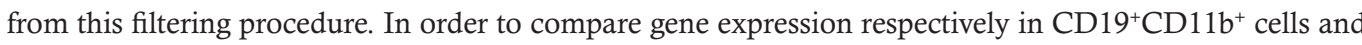
$\mathrm{CD} 19^{+} \mathrm{CD} 11 \mathrm{~b}^{-}$cells across untreated, DT-injured, and DT-injured + pirfenidone conditions, gene expression data were corrected for variability among experimental batches and a principal components analysis across all 12,097 mRNAs for the 17 sequenced samples was performed. This highlighted 5 samples (1 $\mathrm{CD} 19^{+} \mathrm{CD} 11 \mathrm{~b}^{+} \mathrm{DT}$ heart, $2 \mathrm{CD} 19^{+} \mathrm{CD} 11 \mathrm{~b}^{-}$naive hearts, and $1 \mathrm{CD}^{-} 9^{+} \mathrm{CD} 11 \mathrm{~b}^{-} \mathrm{DT}$ heart) that were strong outliers being outside the $95 \%$ confidence interval (2 standard deviations) for their group and that were removed from further analysis. The data are available in the NCBI's Gene Expression Omnibus repository (GEO GSE112984). The limma-voom procedure was used to identify mRNAs with differential expression between experimental conditions (42). KEGG pathway analysis was performed on mRNAs with greater than 2-fold change in expression level between specific experimental groups using the appropriate functional annotation module within the 2017 release of the NIH online resource DAVID (43) and accepting an FDR $\leq 0.05$ (Benjamini-Hochberg method) to identify pathways enriched between the assigned conditions. KEGG pathways within the following 3 categories were analyzed: signal transduction (3.2), signaling molecules and interactions (3.3), and immune system (5.1).

\section{Statistics}

Data are expressed as mean \pm standard deviation. Two-tailed Student's $t$ test was used for pairwise comparisons between 2 groups, 1-way ANOVA with Tukey's correction for multiple post hoc comparisons was used to compare multiple experimental groups, while 2-way ANOVA with Tukey's correction for multiple post hoc comparisons was used to compare across multiple experimental groups with multiple conditions in each group. The Gehan-Breslow-Wilcoxon method was used to compare Kaplan-Meier curves. A $P$ value less than 0.05 was considered statistically significant. The specific statistical test used in each experiment is indicated in the figure captions. All calculations were made using GraphPad Prism version 7.04.

\section{Study approval}

All studies were performed with the approval of the Institutional Animal Care and Use Committee at Washington University School of Medicine. These investigations conform to the NIH Guide for the Care and Use of Laboratory Animals.

\section{Author contributions}

LA designed experiments, performed experiments, and wrote the manuscript. DLM designed experiments and wrote the manuscript. PMB, JDS, GB, CRR, WJ, and DB designed experiments. LJS designed and performed experiments. SJM analyzed the output of whole-genome RNA-sequencing analyses. CJW performed ischemia reperfusion injury on studied animals. AK performed echocardiographic analysis on studied animals. All authors edited and approved the manuscript.

\section{Acknowledgments}

This research was partially funded through NIH grant R01HL107594-06. We thank Erica Lantelme and the Washington University Flow Cytometry \& Fluorescence Activated Cell Sorting Core for assistance provided with flow cytometric analysis and cell sorting. Luigi Adamo was supported by grant T32 HL007081 from the NIH.

Address correspondence to: Douglas L. Mann, Division of Cardiology, 660 S. Euclid Avenue, Campus Box 8086, St. Louis, Missouri 63110, USA. Phone: 314.362.8908; Email: dmann@wustl.edu. 
1. Mann DL. The emerging role of innate immunity in the heart and vascular system: for whom the cell tolls. Circ Res. 2011;108(9):1133-1145.

2. Rymer JA, Newby LK. Failure to launch: Targeting inflammation in acute coronary syndromes. JACC: Basic to Translational Science. 2017;2(4):484-497.

3. Carter NJ. Pirfenidone: in idiopathic pulmonary fibrosis. Drugs. 2011;71(13):1721-1732.

4. Wang Y, Wu Y, Chen J, Zhao S, Li H. Pirfenidone attenuates cardiac fibrosis in a mouse model of TAC-induced left ventricular remodeling by suppressing NLRP3 inflammasome formation. Cardiology. 2013;126(1):1-11.

5. Yamagami K, et al. Pirfenidone exhibits cardioprotective effects by regulating myocardial fibrosis and vascular permeability in pressure-overloaded hearts. Am J Physiol Heart Circ Physiol. 2015;309(3):H512-H522.

6. Yamazaki T, et al. The antifibrotic agent pirfenidone inhibits angiotensin II-induced cardiac hypertrophy in mice. Hypertens Res. 2012;35(1):34-40

7. Mirkovic S, et al. Attenuation of cardiac fibrosis by pirfenidone and amiloride in DOCA-salt hypertensive rats. Br J Pharmacol. 2002;135(4):961-968

8. Van Erp C, Irwin NG, Hoey AJ. Long-term administration of pirfenidone improves cardiac function in mdx mice. Muscle Nerve. 2006;34(3):327-334

9. Miric G, Dallemagne C, Endre Z, Margolin S, Taylor SM, Brown L. Reversal of cardiac and renal fibrosis by pirfenidone and spironolactone in streptozotocin-diabetic rats. Br J Pharmacol. 2001;133(5):687-694.

10. Lee KW, et al. Pirfenidone prevents the development of a vulnerable substrate for atrial fibrillation in a canine model of heart failure. Circulation. 2006;114(16):1703-1712.

11. Nguyen DT, Ding C, Wilson E, Marcus GM, Olgin JE. Pirfenidone mitigates left ventricular fibrosis and dysfunction after myocardial infarction and reduces arrhythmias. Heart Rhythm. 2010;7(10):1438-1445.

12. Li C, et al. Pirfenidone controls the feedback loop of the AT1R/p38 MAPK/renin-angiotensin system axis by regulating liver X receptor- $\alpha$ in myocardial infarction-induced cardiac fibrosis. Sci Rep. 2017;7:40523.

13. Lavine KJ, et al. Distinct macrophage lineages contribute to disparate patterns of cardiac recovery and remodeling in the neonatal and adult heart. Proc Natl Acad Sci USA. 2014;111(45):16029-16034.

14. Epelman S, et al. Embryonic and adult-derived resident cardiac macrophages are maintained through distinct mechanisms at steady state and during inflammation. Immunity. 2014;40(1):91-104.

15. Lavine KJ, Kovacs A, Weinheimer C, Mann DL. Repetitive myocardial ischemia promotes coronary growth in the adult mammalian heart. J Am Heart Assoc. 2013;2(5):e000343.

16. Kantor AB, Stall AM, Adams S, Herzenberg LA, Herzenberg LA. Differential development of progenitor activity for three B-cell lineages. Proc Natl Acad Sci USA. 1992;89(8):3320-3324.

17. Rahman ZS. Impaired clearance of apoptotic cells in germinal centers: implications for loss of B cell tolerance and induction of autoimmunity. Immunol Res. 2011;51(2-3):125-133.

18. Zhang W, et al. Necrotic myocardial cells release damage-associated molecular patterns that provoke fibroblast activation in vitro and trigger myocardial inflammation and fibrosis in vivo. J Am Heart Assoc. 2015;4(6):e001993.

19. Xu H, Liew LN, Kuo IC, Huang CH, Goh DL, Chua KY. The modulatory effects of lipopolysaccharide-stimulated B cells on differential T-cell polarization. Immunology. 2008;125(2):218-228.

20. Bönner F, Borg N, Burghoff S, Schrader J. Resident cardiac immune cells and expression of the ectonucleotidase enzymes CD39 and CD73 after ischemic injury. PLoS ONE. 2012;7(4):e34730.

21. Ramos GC, et al. Myocardial aging as a T-cell-mediated phenomenon. Proc Natl Acad Sci USA. 2017;114(12):E2420-E2429.

22. Yan X, et al. Temporal dynamics of cardiac immune cell accumulation following acute myocardial infarction. J Mol Cell Cardiol. 2013;62:24-35.

23. Zouggari Y, et al. B lymphocytes trigger monocyte mobilization and impair heart function after acute myocardial infarction. Nat Med. 2013;19(10):1273-1280.

24. Horckmans M, et al. Pericardial adipose tissue regulates granulopoiesis, fibrosis, and cardiac function after myocardial infarction. Circulation. 2018;137(9):948-960.

25. Cordero-Reyes AM, et al. Full expression of cardiomyopathy is partly dependent on B-cells: a pathway that involves cytokine activation, immunoglobulin deposition, and activation of apoptosis. J Am Heart Assoc. 2016;5(1):e002484.

26. Zhang M, Michael LH, Grosjean SA, Kelly RA, Carroll MC, Entman ML. The role of natural IgM in myocardial ischemia-reperfusion injury. J Mol Cell Cardiol. 2006;41(1):62-67.

27. Shen P, Fillatreau S. Antibody-independent functions of B cells: a focus on cytokines. Nat Rev Immunol. 2015;15(7):441-451

28. Rubtsova K, Rubtsov AV, Cancro MP, Marrack P. Age-associated B cells: A T-bet-dependent effector with roles in protective and pathogenic immunity. J Immunol. 2015;195(5):1933-1937.

29. Hua Z, Hou B. TLR signaling in B-cell development and activation. Cell Mol Immunol. 2013;10(2):103-106.

30. Yamamoto M, et al. Essential role for TIRAP in activation of the signalling cascade shared by TLR2 and TLR4. Nature 2002;420(6913):324-329.

31. Bizargity P, Liu K, Wang L, Hancock WW, Visner GA. Inhibitory effects of pirfenidone on dendritic cells and lung allograft rejection. Transplantation. 2012;94(2):114-122.

32. Mann DL. Innate immunity and the failing heart: the cytokine hypothesis revisited. Circ Res. 2015;116(7):1254-1268.

33. Nossuli TO, et al. A chronic mouse model of myocardial ischemia-reperfusion: essential in cytokine studies. Am J Physiol Heart Circ Physiol. 2000;278(4):H1049-H1055.

34. Burchfield JS, et al. The cytoprotective effects of tumor necrosis factor are conveyed through tumor necrosis factor receptor-associated factor 2 in the heart. Circ Heart Fail. 2010;3(1):157-164.

35. Tzeng HP, et al. Dysferlin mediates the cytoprotective effects of TRAF2 following myocardial ischemia reperfusion injury. $J$ Am Heart Assoc. 2014;3(1):e000662.

36. Schaefer CJ, Ruhrmund DW, Pan L, Seiwert SD, Kossen K. Antifibrotic activities of pirfenidone in animal models. Eur Respir Rev. 2011;20(120):85-97.

37. Lavine KJ, et al. Coronary collaterals predict improved survival and allograft function in patients with coronary allograft vascu- 
lopathy. Circ Heart Fail. 2013;6(4):773-784.

38. Kanno S, et al. Echocardiographic evaluation of ventricular remodeling in a mouse model of myocardial infarction. $J A m$ Soc Echocardiogr. 2002;15(6):601-609.

39. Misharin AV, Saber R, Perlman H. Eosinophil contamination of thioglycollate-elicited peritoneal macrophage cultures skews the functional readouts of in vitro assays. J Leukoc Biol. 2012;92(2):325-331.

40. Trapnell C, Pachter L, Salzberg SL. TopHat: discovering splice junctions with RNA-Seq. Bioinformatics. 2009;25(9):1105-1111.

41. Anders S, Pyl PT, Huber W. HTSeq--a Python framework to work with high-throughput sequencing data. Bioinformatics. 2015;31(2):166-169.

42. Zhou X, Lindsay H, Robinson MD. Robustly detecting differential expression in RNA sequencing data using observation weights. Nucleic Acids Res. 2014;42(11):e91.

43. Huang da W, Sherman BT, Lempicki RA. Systematic and integrative analysis of large gene lists using DAVID bioinformatics resources. Nat Protoc. 2009;4(1):44-57. 